\title{
Systematic genetic mapping of necroptosis identifies SLC39A7 as modulator of death receptor trafficking
}

\author{
Astrid Fauster $\mathbb{D}^{1} \cdot$ Manuele Rebsamen $\mathbb{1}^{1} \cdot$ Katharina L. Willmann $\mathbb{1}^{1,2} \cdot$ Adrian César-Razquin $^{1} \cdot$ Enrico Girardi $^{1}$. \\ Johannes W. Bigenzahn ${ }^{1}$ • Fiorella Schischlik ${ }^{1}$ - Stefania Scorzoni ${ }^{1}$ - Manuela Bruckner ${ }^{1}$. Justyna Konecka ${ }^{1}$.

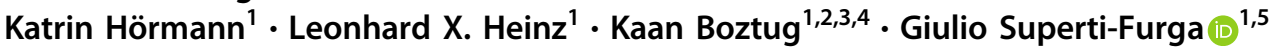

Received: 26 March 2018 / Revised: 4 July 2018 / Accepted: 22 July 2018 / Published online: 20 September 2018

(c) The Author(s) 2018. This article is published with open access

\begin{abstract}
Regulation of cell and tissue homeostasis by programmed cell death is a fundamental process with wide physiological and pathological implications. The advent of scalable somatic cell genetic technologies creates the opportunity to functionally map such essential pathways, thereby identifying potential disease-relevant components. We investigated the genetic basis underlying necroptotic cell death by performing a complementary set of loss-of-function and gain-of-function genetic screens. To this end, we established $F A D D$-deficient haploid human KBM7 cells, which specifically and efficiently undergo necroptosis after a single treatment with either TNF $\alpha$ or the SMAC mimetic compound birinapant. A series of unbiased gene-trap screens identified key signaling mediators, such as TNFR1, RIPK1, RIPK3, and MLKL. Among the novel components, we focused on the zinc transporter SLC39A7, whose knock-out led to necroptosis resistance by affecting TNF receptor surface levels. Orthogonal, solute carrier (SLC)-focused CRISPR/Cas9-based genetic screens revealed the exquisite specificity of SLC39A7, among 400 SLC genes, for TNFR1-mediated and FAS-mediated but not TRAIL-R1-mediated responses. Mechanistically, we demonstrate that loss of SLC39A7 resulted in augmented ER stress and impaired receptor trafficking, thereby globally affecting downstream signaling. The newly established cellular model also allowed genomewide gain-of-function screening for genes conferring resistance to necroptosis via the CRISPR/Cas9-based synergistic activation mediator approach. Among these, we found cIAP1 and cIAP2, and characterized the role of TNIP1, which prevented pathway activation in a ubiquitin-binding dependent manner. Altogether, the gain-of-function and loss-of-function screens described here provide a global genetic chart of the molecular factors involved in necroptosis and death receptor signaling, prompting further investigation of their individual contribution and potential role in pathological conditions.
\end{abstract}

Edited by S. Nagata

These authors contributed equally: Astrid Fauster, Manuele Rebsamen

Electronic supplementary material The online version of this article (https://doi.org/10.1038/s41418-018-0192-6) contains supplementary material, which is available to authorized users.

Manuele Rebsamen

mrebsamen@cemm.oeaw.ac.at

$\triangle$ Giulio Superti-Furga

gsuperti@cemm.oeaw.ac.at

1 CeMM Research Center for Molecular Medicine of the Austrian Academy of Sciences, 1090 Vienna, Austria

2 Ludwig Boltzmann Institute for Rare and Undiagnosed Diseases,

\section{Introduction}

Regulated cell death programs are crucial for homeostasis in multicellular organisms by eliminating cells that have become obsolete, damaged or infected [1]. Cell death signaling can be induced extrinsically by death receptors of the TNF receptor superfamily, including tumor necrosis factor receptor superfamily member $1 \mathrm{~A}$ (TNFR1), Fas cell surface

1090 Vienna, Austria

3 Department of Pediatrics and Adolescent Medicine, Medical University of Vienna, 1090 Vienna, Austria

4 Department of Pediatrics, St. Anna Kinderspital and Children's Cancer Research Institute, Medical University of Vienna, 1090 Vienna, Austria

5 Center for Physiology and Pharmacology, Medical University of Vienna, Vienna, Austria 
death receptor (FAS), TNF-related apoptosis-inducing ligand receptor (TRAIL-R)1 and 2 [2]. TNFR1 stimulation can lead to NF-KB activation and survival, apoptosis, or necroptosis depending on the composition of the signal transduction complexes formed upon ligand binding [2]. Necroptosis can be triggered by different death and immune receptors in response to pathogen infection or in the context of sterile inflammation [2-4], and relies on activation of receptor-interacting serine/threonine-protein kinase (RIPK) 3 [5-7] and its substrate mixed lineage kinase domain-like protein (MLKL) $[8,9]$, which mediates membrane rupture. Death receptor ligation triggers RIPK3 activation through RIPK1, in conditions where apoptosis is blocked [5, 10, 11]. Evidence for the involvement of necroptosis in different pathologies [4, 12-15], such as ischemia/reperfusionmediated injuries [16], has accumulated over the past years. While exacerbating these inflammatory conditions, necroptosis can be beneficial in other pathological contexts, particularly in restraining viral and bacterial infections $[4,17]$. Consequently, detailed insight into the molecular framework of necroptotic cell death entails the appealing prospect of therapeutic benefit $[12,13]$.

Forward genetics with somatic cells constitutes a powerful, unbiased approach to unravel the genetic basis underlying fundamental biological processes. RNA interference approaches led to the identification of the core necroptosis pathway members RIPK3 and MLKL [5, 6, 9]. By allowing efficient generation of full knockouts through insertional mutagenesis, the near-haploid KBM7 cell line has further empowered screening approaches in human cells $[18,19]$. The scope of genetic screening has been broadened by the advent of CRISPR/Cas 9 technology and its adaptation to gain-of-function screening modes, such as the development of synergistic activation mediator (SAM) libraries mediating transcriptional activation of endogenous genes [20-22]. In this study, we combine these technologies to investigate the genetic foundation of $\mathrm{TNF} \alpha$-induced necroptosis and provide a comprehensive mapping of the molecular factors controlling necroptosis signaling. We characterize the specific contributions of the zinc transporter SLC39A7 by demonstrating its requirement for death receptor trafficking, thereby affecting all aspects of TNFR1 signaling, and of the ubiquitin-engaging protein TNIP1 on necroptosis pathway activation.

\section{Results}

\section{A KBM7 FADD cell line undergoes necroptosis upon treatment with TNFa or the SMAC mimetic birinapant}

We set out to map the genetic requirements for necroptosis signaling in human cells, employing the haploid myeloid leukemia KBM7 cell line $[18,19]$. In contrast to the related HAP1 cell line that lacks RIPK3 expression [23], KBM7 undergo necroptosis upon treatment with TNF $\alpha$, the SMAC mimetic birinapant [24] and the pan-caspase inhibitor z-VAD-FMK (Fig. 1a, Supplementary Figure 1a). As apoptosis inhibition is required for death receptorinduced necroptosis [25], we genetically abrogated the extrinsic apoptosis pathway by deleting the signaling adapter Fas associated via death domain (FADD) by CRISPR/Cas 9 gene editing (Supplementary Figure 1b-c). After enrichment for resistance to FASL-induced and TRAIL-induced apoptosis, we selected a knockout clone carrying a $>100 \mathrm{bp}$ insertion in the sgRNA target site, abrogating FADD expression (Supplementary Figure 1c-e). As expected, absence of FADD did not affect TNF $\alpha$ induced NF- $\mathrm{\kappa B}$ activation (Supplementary Figure 1f). Necroptosis could be induced in KBM7 FADD cells using $\mathrm{TNF} \alpha$ or a combination of $\mathrm{TNF} \alpha$ and SMAC mimetic without requirement for caspase inhibition, and was blocked by the RIPK1 inhibitor Nec1-s [26] or by blocking MLKL using necrosulfonamide (NSA) [8], whereas z-VAD-FMK had no effect (Fig. 1a, b). Indeed, treatment with TNF $\alpha$ and SMAC mimetic triggered rapid phosphorylation of MLKL in KBM7 $F A D D^{-}$cells, whereas it induced apoptosis in KBM7 wildtype cells, as evidenced by Caspase-3 cleavage (Supplementary Figure 1g). Interestingly, treatment with the SMAC mimetic birinapant alone sufficed to induce necroptosis in KBM7 FADD cells (Fig. 1a). In the following, these newly established $F A D D$-deficient KMB7 cells were used to interrogate the genetic basis of necroptotic cell death by employing complementary forward genetics approaches (Supplementary Figure 1h).

\section{Haploid genetic screens in KBM7 FADD- cells identify the requirements for necroptosis}

In order to identify genes required for necroptosis signaling by haploid genetic screening, KBM7 FADD cells were mutagenized with a retroviral gene-trap vector $[18,19]$ and selected with a high dose of the SMAC mimetic birinapant, TNF $\alpha$, or a combination thereof. Each of these screens resulted in significant ( $p$-value $<10^{-10}$ ) enrichment of disruptive insertions in 10-13 different genes compared to unselected mutagenized cells (Fig. 2a-c, Supplementary Table 1). The screens identified TNFRSF1A, RIPKI, RIPK3, and MLKL among the top hits with a high number of independent insertions, consistent with their wellestablished role in TNF $\alpha$-induced necroptosis signaling and a recent loss-of-function screen in murine cells [27] (Fig. 2d, Supplementary Figure 2a,b). Interestingly, alongwith these known necroptosis effector proteins, the zinc transporter SLC39A7 scored among the most significant 

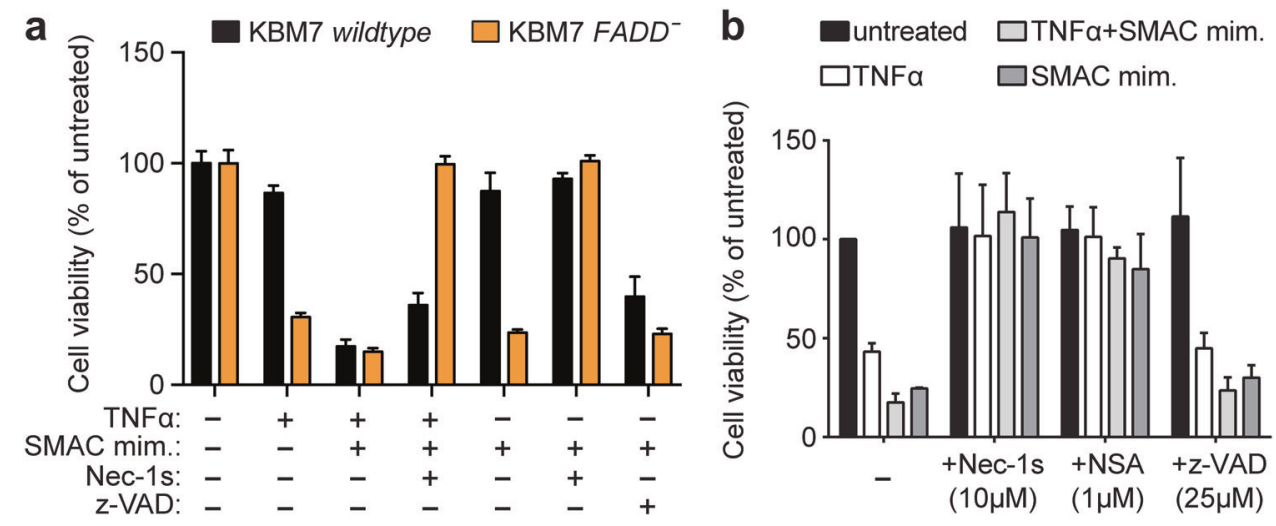

Fig. $1 \mathrm{KBM} 7 F A D D^{-}$cells undergo necroptosis upon treatment with $\mathrm{TNF} \alpha$ or the SMAC mimetic birinapant. a Cell viability of KMB7 wildtype and KBM7 FADD cells treated for $24 \mathrm{~h}$ with $100 \mathrm{ng} / \mathrm{ml}$ $\mathrm{TNF} \alpha, 1 \mu \mathrm{M}$ SMAC mimetic birinapant, $50 \mu \mathrm{M}$ Nec-1s and $10 \mu \mathrm{M}$ zVAD-FMK as indicated. b Cell viability of KBM7 $F A D D^{-}$cells treated for $24 \mathrm{~h}$ with $100 \mathrm{ng} / \mathrm{ml} \mathrm{TNF} \alpha, 10 \mu \mathrm{M}$ SMAC mimetic or $100 \mathrm{ng} / \mathrm{ml}$
TNF $\alpha$ and $1 \mu \mathrm{M}$ SMAC mimetic, and the indicated inhibitors. Cell viability was assessed using a luminescence-based readout for ATP (CellTiter Glo) and normalized to untreated control. Data represent mean value \pm s.d. of two independent experiments performed in triplicates hits in all screens, while other genes significantly enriched in selected conditions, such as Tumor necrosis factor receptor superfamily member $1 \mathrm{~B} \quad(T N F R S F 1 B)$ and Sp1 (SP1) with the SMAC mimetic birinapant (Fig. 2d, Supplementary Figure 2c). For follow-up analyses, we focused on the highly or selectively enriched genes, employing a CRISPR/Cas9-based multi-color competition assay (MCA) co-culture system for validation (Supplementary Figure 2d). Both SMAC mimetic as well as TNF $\alpha$ treatment strongly selected for $\mathrm{GFP}^{+} \operatorname{sgSLC} 39 \mathrm{A7}^{-}$harboring cells over control mCherry ${ }^{+}$cells harboring $\mathrm{sgRen}$ (targeting Renilla luciferase), indicating that loss of SLC39A7 conferred enhanced cell survival or outgrowth under necroptosis-inducing conditions (Fig. 2e). Among the other genes tested, we confirmed the selective advantage upon treatment with the SMAC mimetic birinapant of cells harboring sgRNAs targeting $S P 1$, TNFRSF1B, and, to a lesser extent, PU.1 (SPII) and Ragulator complex protein LAMTOR1 (LAMTOR1) (Fig. 2f, Supplementary Figure 2e).

\section{Loss of SLC39A7 mediates resistance to TNFa- induced cell death by diminishing TNFR1 surface expression}

Next, we investigated how loss of SLC39A7 impacts on TNF $\alpha$ signaling, given that the proposed roles for this ER-resident zinc transporter did not readily explain its link to the necroptosis phenotype [28-32]. We isolated a KBM7 $F A D D^{-}$clone carrying a $5 \mathrm{bp}$ deletion in the SLC39A7 coding sequence, leading to a premature stop codon and loss of protein expression (Supplementary Figure 3a). SLC39A7 has been reported as an essential gene in a number of cell lines of different tissue origin [33, 34], but does not form part of the core essentialome in KBM7 cells [35]. Yet, loss of SLC39A7 conferred a significant growth disadvantage as compared to sgRen control cells (Fig. 3a). In agreement with our screening results, SLC39A7 knockout cells were protected from TNFo and SMAC mimetic-induced necroptosis (Fig. 3b). Strikingly, SLC39A7 cells failed to activate the canonical NF-KB pathway following TNF $\alpha$ stimulation, and this correlated with the loss of TNFR1 surface expression (Fig. 3c, d). We confirmed that SLC39A7 localizes to the ER [28] (Supplementary Figure $3 b-c$ ) and, to assess the cellular alterations resulting from its loss, we determined changes in protein levels by employing a proteomics approach focused on membrane compartments (Supplementary Figure 3d-e). Gene ontology (GO) term enrichment among the proteins significantly upregulated in $S L C 39 A 7^{-}$cells and gene set enrichment analysis (GSEA) among all significantly changed targets respectively identified "response to endoplasmic reticulum stress" and the hallmark gene set "Unfolded_protein_response" (UPR) as top hits (Supplementary Figure 3f-h, Supplementary Table 2). These data strongly indicated that, similar to its Drosophila and murine orthologues [30, 31, 36], loss of human SLC39A7 impacts on ER homeostasis. Indeed, SLC 39A7 cells displayed ER stress at basal state, reflected in increased levels of $\mathrm{BiP}$ (ER chaperone $78 \mathrm{kDa}$ glucose-regulated protein GRP), and exhibited higher sensitivity towards different chemical ER stressors as monitored by induction of CHOP (DNA damage-inducible transcript 3 protein DDIT3) (Fig. 3e). Given the crucial role of the ER in the synthesis, folding and shuttling of membrane proteins [37], we next explored how loss of SLC39A7 impinged on TNFR1 

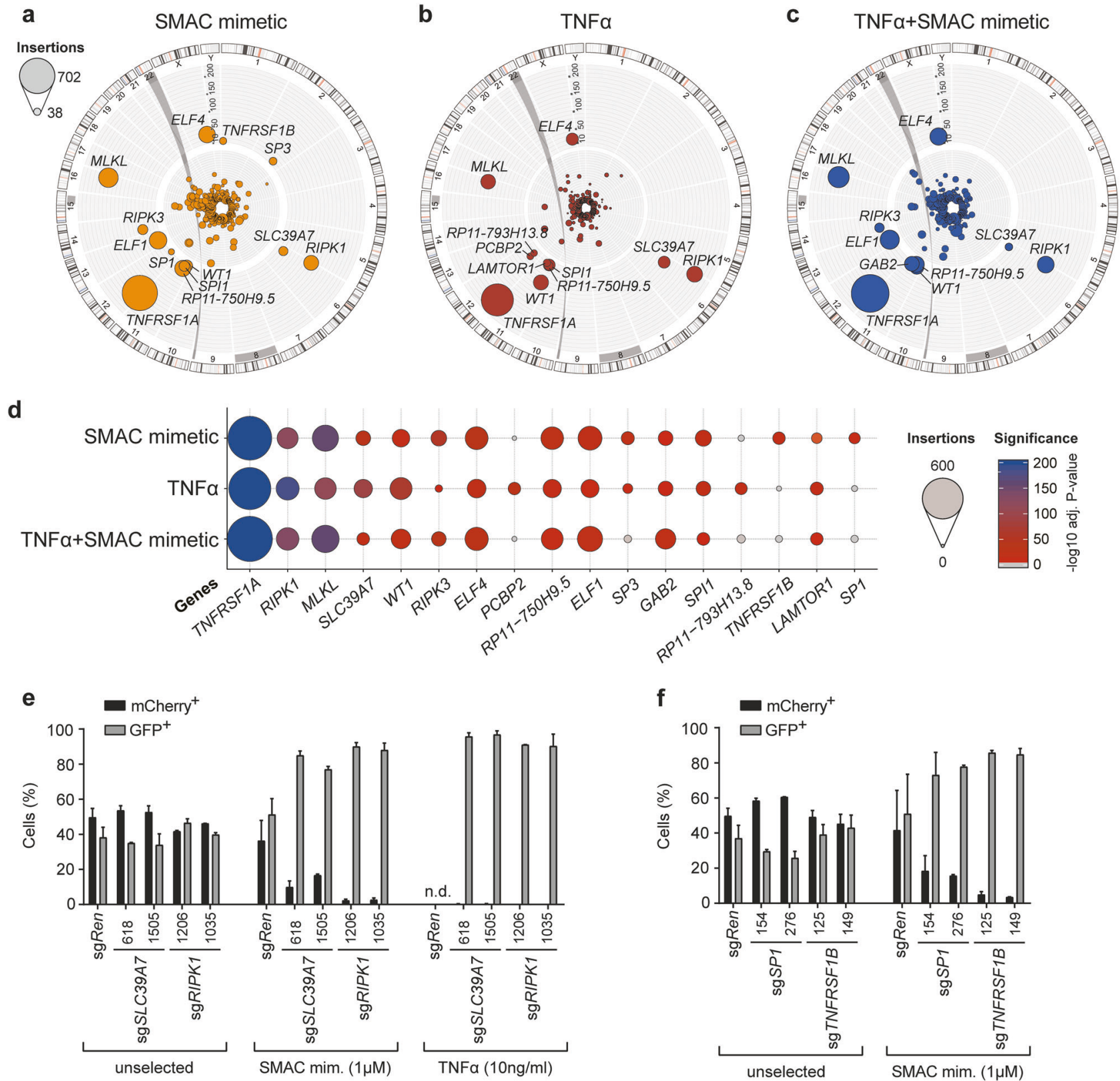

Fig. 2 Haploid genetic screens in KBM7 $F A D D^{-}$cells identify genes required for necroptosis. a-c Circos plots of haploid genetic screens in KBM7 FADD cells with necroptosis induction by $10 \mu \mathrm{M}$ SMAC mimetic birinapant (a) $100 \mathrm{ng} / \mathrm{ml} \mathrm{TNF} \alpha$ (b) and $1 \mu \mathrm{M}$ SMAC mimetic and $100 \mathrm{ng} / \mathrm{ml} \mathrm{TNF} \alpha$ combined (c). Each dot represents a mutagenized gene identified in the resistant cell population, dot size corresponds to the number of independent insertions identified for each gene and distance from center indicates the significance of enrichment compared to an unselected control data set. Hits with an adjusted $p$-value $<10^{-10}$ are labeled. d Bubble plot depicting the top hits over all three screens ranked according to adjusted $p$-value in the TNF $\alpha$ screen. Bubble size

trafficking. Surprisingly, while TNFR1 was not detectable on the surface of $S L C 39 A 7^{-}$cells (Fig. 3d), we found higher levels of intracellular TNFR1 protein in SLC39A7 knockouts (Fig. 3f). Using glycan maturation as readout for corresponds to the number of independent insertions identified and color gradient reflects the significance of enrichment. e, f Multi-color competition assay (MCA) of KBM7 FADD ${ }^{-} \operatorname{SpCas} 9$ cells transduced with a GFP marker $\left(\mathrm{GFP}^{+}\right)$and sgRNAs targeting either $S L C 39 A 7$ or RIPK1 (e), SP1 or TNFR2 (f), or Renilla luciferase (sgRen) as control, against cells transduced with $\mathrm{sgRen}$ and an mCherry marker $\left(\mathrm{mCherry}^{+}\right)$. The cell populations were mixed at 1:1 ratio, treated with SMAC mimetic $(1 \mu \mathrm{M})$ or $\mathrm{TNF} \alpha(10 \mathrm{ng} / \mathrm{ml})$, and analyzed after 14 days by flow cytometry. Data represent mean value \pm s.d. of two independent experiments performed in duplicates, n.d. (not determined) indicates wells with no outgrowth

glycoprotein movement through the secretory pathway [38], we found that TNFR1 accumulating in SLC39A7 cells exhibited sensitivity to Endo $\mathrm{H}$, indicative of retained ER localization (Fig. 3g). 


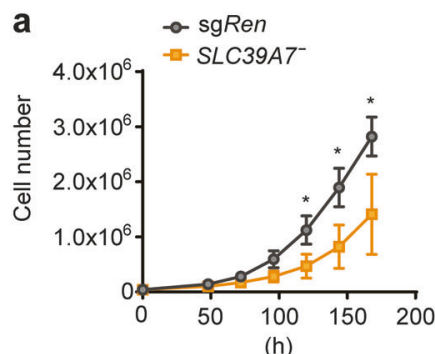

(h)

C
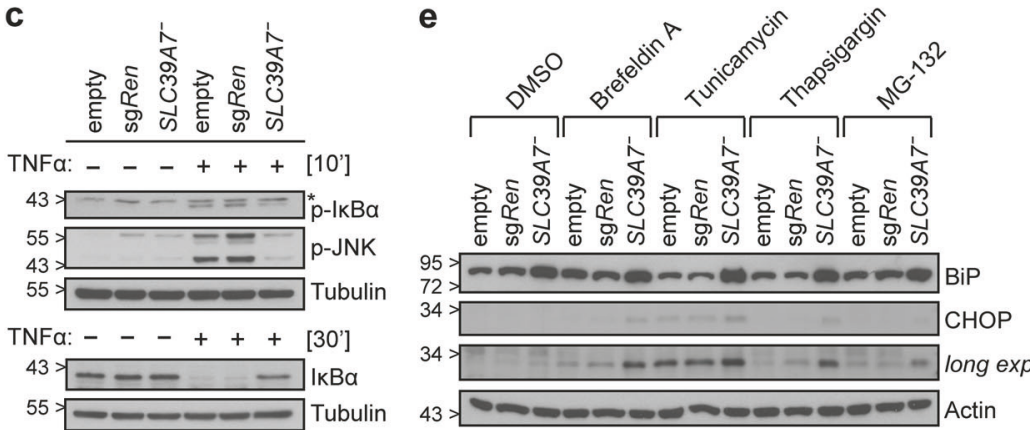

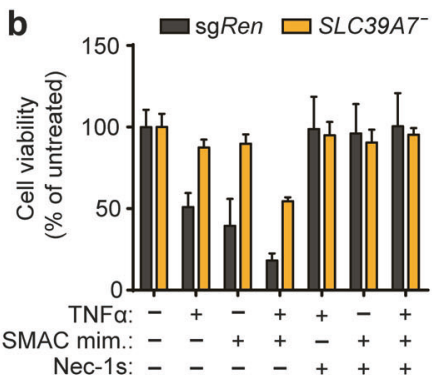

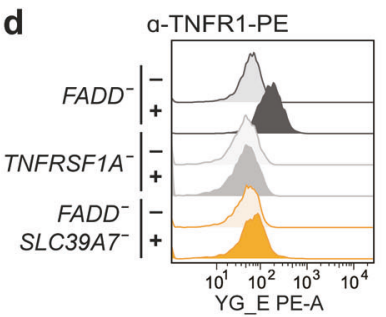

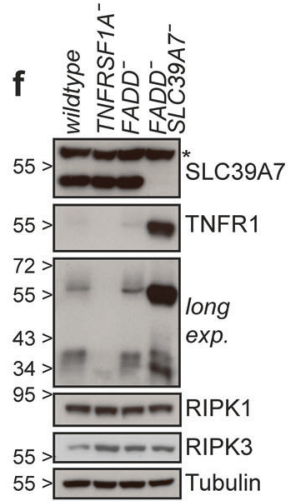

55
Fig. 3 Loss of SLC39A7 mediates resistance to TNF $\alpha$-induced cell death by diminishing TNFR1 surface expression. a Growth curve for KBM7 FADD ${ }^{-} \operatorname{sgRen}$ or $S L C 39 A 7^{-}$cells. Equal cell numbers were seeded and cell growth monitored over 7 days. Data represent mean value \pm s.d. of five independent experiments; statistical analysis by $t$ test, $* P<0.01$. b Cell viability in KBM7 $F A D D^{-} \operatorname{sgRen}$ or $S L C 39 A 7^{-}$ cells treated overnight $(16 \mathrm{~h})$ with $\mathrm{TNF} \alpha(10 \mathrm{ng} / \mathrm{ml})$, SMAC mimetic $(0.5 \mu \mathrm{M})$, and Nec-1s $(50 \mu \mathrm{M})$ as indicated. Cell viability was assessed using a luminescence-based readout for ATP (CellTiter Glo). Data represent mean value \pm s.d. of two independent experiments performed in triplicates. c KBM7 FADD ${ }^{-}$SpCas9 (empty, sgRen or SLC39A7) cells were stimulated for the time indicated with $\mathrm{TNF} \alpha(10 \mathrm{ng} / \mathrm{ml})$. Cells were then lysed and subjected to immunoblotting with the indicated antibodies. d Flow cytometry analysis for TNFR1 surface expression. KBM7 TNFRSF1A cells serve as negative control for background staining. Data shown are representative of two independent experiments. e KBM7 FADD SpCas9 (empty, sgRen or $\left.S L C 39 A^{-}\right)$cells were treated for $7 \mathrm{~h}$ with Brefeldin A $(0.5 \mu \mathrm{M})$, Tunicamycin $(2 \mu \mathrm{M})$, Thapsigargin $(0.5 \mu \mathrm{M})$, MG-132 $(10 \mu \mathrm{M})$ or DMSO as control. Cells were then lysed and subjected to immunoblotting with the indicated antibodies. f KBM7 TNFRSF1A- KBM7 $F A D D^{-}$and KBM7 FADD $S L C 39 A 7^{-}$cells were lysed and subjected to immunoblotting with the indicated antibodies. g KBM7 $F A D D^{-}$ SpCas $9 \mathrm{sgRen}$ or $S L C 39 A 7^{-}$cell lysates were incubated for $1 \mathrm{~h}$ at $37^{\circ} \mathrm{C}$ in presence or absence of PNGaseF or EndoH, respectively, and analysed by immunoblot with the indicated antibodies. Immunoblots shown are representative of two independent experiments. Asterisk (*) indicates non-specific band

\section{Orthogonal genetic screens and surface marker analysis define the specificity of SLC39A7 on receptor trafficking}

To study the specificity of the SLC39A7 loss on death receptor trafficking, we devised a set of targeted and genome-wide screens including the other prominent TNF receptor superfamily members, FAS and TRAIL-R1/2. The use of a focused CRISPR/Cas 9 library targeting 388 members of the SLC family allowed us to efficiently screen various conditions and stimuli in multiple cell lines. KBM7 $F A D D^{-}, \mathrm{KBM} 7$ wildtype and HAP1 cells were lentivirally transduced with the library and subsequently selected for resistance towards cell death induction by TNF $\alpha$, SMAC mimetic, TRAIL, or FASL (Supplementary Figure 4a). sgRNAs targeting SLC39A7 were top-enriched in screens with TNF $\alpha$, SMAC mimetic, and, interestingly, FASL (Fig. 4a, Supplementary Table 4 and 5), highlighting the exquisite role of SLC39A7 among SLCs in affecting these signaling pathways. Intriguingly, loss of SLC39A7 did not confer a selective advantage when TRAIL was used to induce apoptotic cell death in KBM7 wildtype and HAP1 cells (Fig. 4a, Supplementary Figure 4b). Genomewide haploid genetic screening in KBM7 cells confirmed the divergent requirement for SLC39A7 between TRAILand FASL-induced cell death (Supplementary Figure 4c, Supplementary Table 1). In line with the screening results, treatment with FASL selected for $\mathrm{GFP}^{+}$sgSLC39A7-harboring cells, whereas we found no differential outgrowth in TRAIL-selected conditions in both Jurkat E6.1 and KBM7 cells (Fig. 4b, Supplementary Figure 4d). Similar to TNFR1, surface expression of FAS and TRAIL-R2 was reduced in SLC39A7 knockout cells, while, in contrast, TRAIL-R1 was still detectable and even slightly increased (Fig. 4c, Supplementary Figure 4e). Accordingly, total and, more importantly, Endo H-resistant levels of TRAIL-R1 showed a comparable increase in SLC39A7 knockout cells, differing therefore from the fully Endo $\mathrm{H}$-sensitive accumulation of TNFR1 in these cells (Supplementary Figure $4 \mathrm{f}$ ). While explaining the retained sensitivity to TRAIL- 


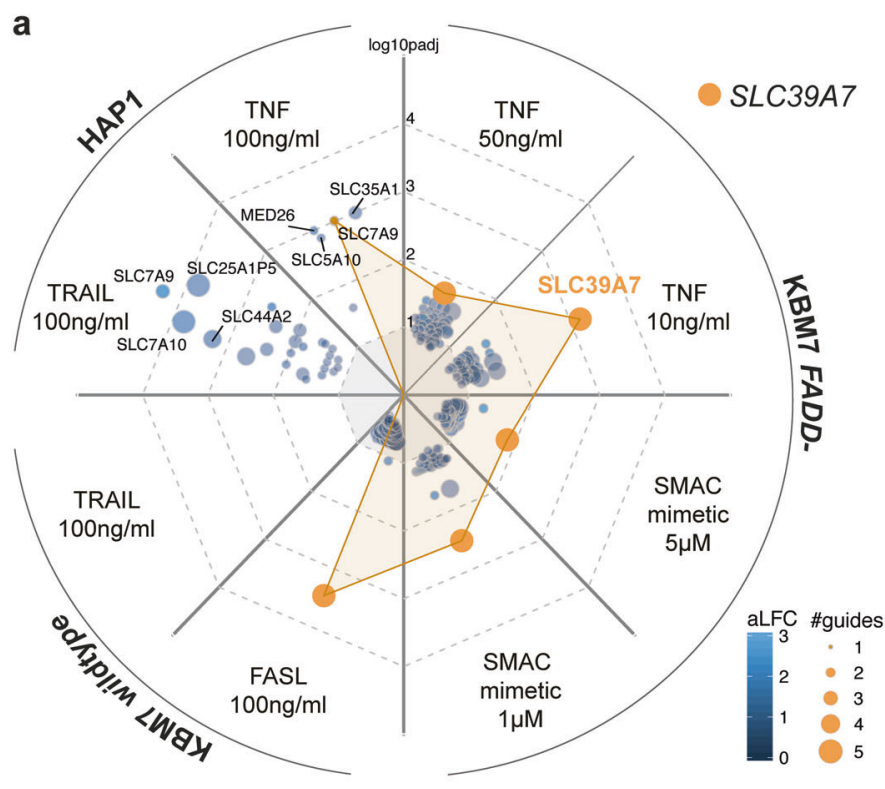

Fig. 4 Orthogonal genetic screens and surface marker analysis specify SLC39A7 loss-of-function phenotype on receptor trafficking. a Spider plot summarizing the results of 8 independent CRISPR/Cas 9 screens in KBM7 wildtype, KBM7 FADD', or HAP1 cells using an SLCspecific gRNA library. Each plot section represents one screen with the indicated stimuli. Screen analysis was performed by identifying differentially enriched sgRNAs using DESeq2 and then aggregating sgRNAs to genes using Gene Set Enrichment Analysis. Identified hits are ranked according to the adjusted $p$-value of enrichment $(-\log 10$ $\left(p_{a d j}\right)$ ), bubble size indicates the number of significantly enriched sgRNAs and color the average $\log 2$ fold-change (aLFC) of the enriched sgRNAs. Screens were performed in duplicate. SLC39A7 is highlighted in orange. No gene was identified in KBM7 wildtype cells

induced cell death, the dichotomy between these two closely related receptors was surprising. Consequently, we monitored a wider panel of cell surface proteins present on KBM7 cells (Supplementary Figure 4g) [39]. Several markers, including CD31, CD11a, CD34, CD45, and CD55 exhibited altered surface expression in SLC39A7 knockout cells, while others, such as CD4, C3AR, or C5L2, remained unchanged. Taken together, these data indicate that specific membrane proteins are differentially affected by loss of SLC39A7.

\section{SLC39A7 transporter function is required for ER homeostasis, TNFR1 surface expression and cell death induction}

To corroborate the link between SLC39A7 loss and the phenotypes observed, we reconstituted KBM7 FADD SLC39A7 knockout cells with V5-tagged SLC39A7 or GFP (Fig. 5a). SLC39A7 reconstitution decreased BiP expression, restored TNFR1 surface levels and, consequently, sensitivity to cell death induction with TNF $\alpha$ and SMAC mimetic (Fig. 5b, c). To investigate the requirement of SLC39A7 transport activity, we performed analogous b

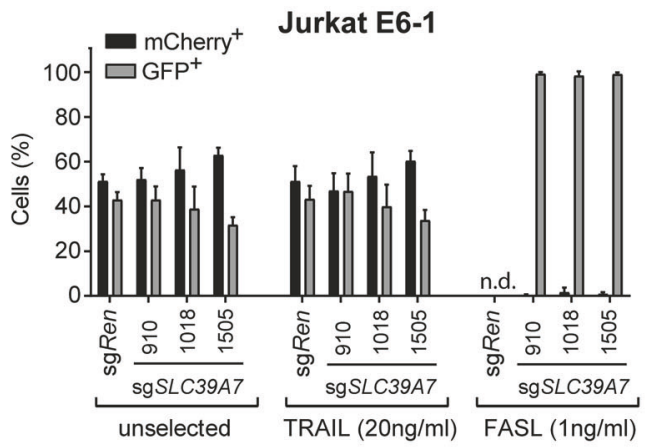

C
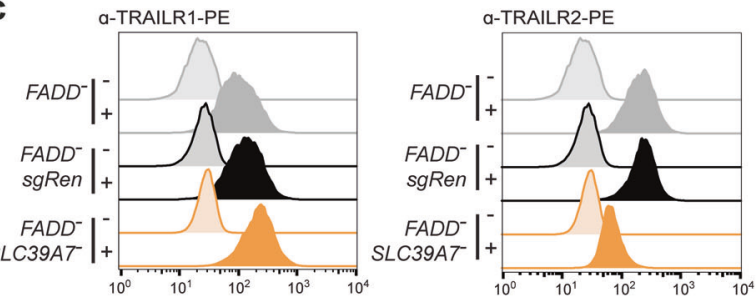

upon TRAIL treatment. b MCA of Jurkat E6.1 SpCas9 cells transduced with a GFP marker $\left(\mathrm{GFP}^{+}\right)$and sgRNAs targeting either SLC39A7 or Renilla luciferase (sgRen) as control, against cells transduced with $\mathrm{sgRen}$ and an mCherry marker $\left(\mathrm{mCherry}^{+}\right)$. The cell populations were mixed at 1:1 ratio, treated with TRAIL $(20 \mathrm{ng} / \mathrm{ml})$ or FASL ( $1 \mathrm{ng} / \mathrm{ml})$, and analyzed after 14 days by flow cytometry. Data represent mean value \pm s.d. of two independent experiments performed in duplicates, n.d. (not determined) indicates wells with no outgrowth. c Flow cytometry analysis for TRAILR1 (left) and TRAILR2 (right) surface expression in KBM7 FADD, KBM7 FADD ${ }^{-} \operatorname{sg}$ Ren or KBM7 $F A D D^{-}$SLC39A7 cells. Data shown are representative of two independent experiments

reconstitution experiments with SLC39A7 constructs bearing substitutions at conserved histidines (H329A or H358A) predicted to participate in an intramembranous zinc-binding site or at other conserved residues (H362A or G365R) within the same metalloprotease-like motif [40]. SLC39A7 H329A and H358A mutants failed to restore sensitivity to necroptosis and to relieve ER stress, whereas H362A and G365R behaved similar to the wildtype construct (Fig. 5d, e). These results are consistent with recent structural and transport data for SLC39A4, in which substitution of histidines corresponding to SLC39A7 H329 and H358, but not H362, was shown to affect transport activity [41]. Compared to the constructs able to functionally rescue SLC39A7 deficiency, H329A and H358A mutants showed reduced expression in KBM7 FADD ${ }^{-}$SLC39A7 cells (Fig. 5e). This is likely a consequence of the unresolved ER stress, as all constructs were expressed at similar level in SLC39A7-proficient cells (Fig. 5e). While an intrinsic reduced stability of H329A and H358A mutants cannot be excluded, their expression was comparable to endogenous SLC39A7 levels, strongly suggesting that transport activity is required to restore ER homeostasis and necroptosis sensitivity. 

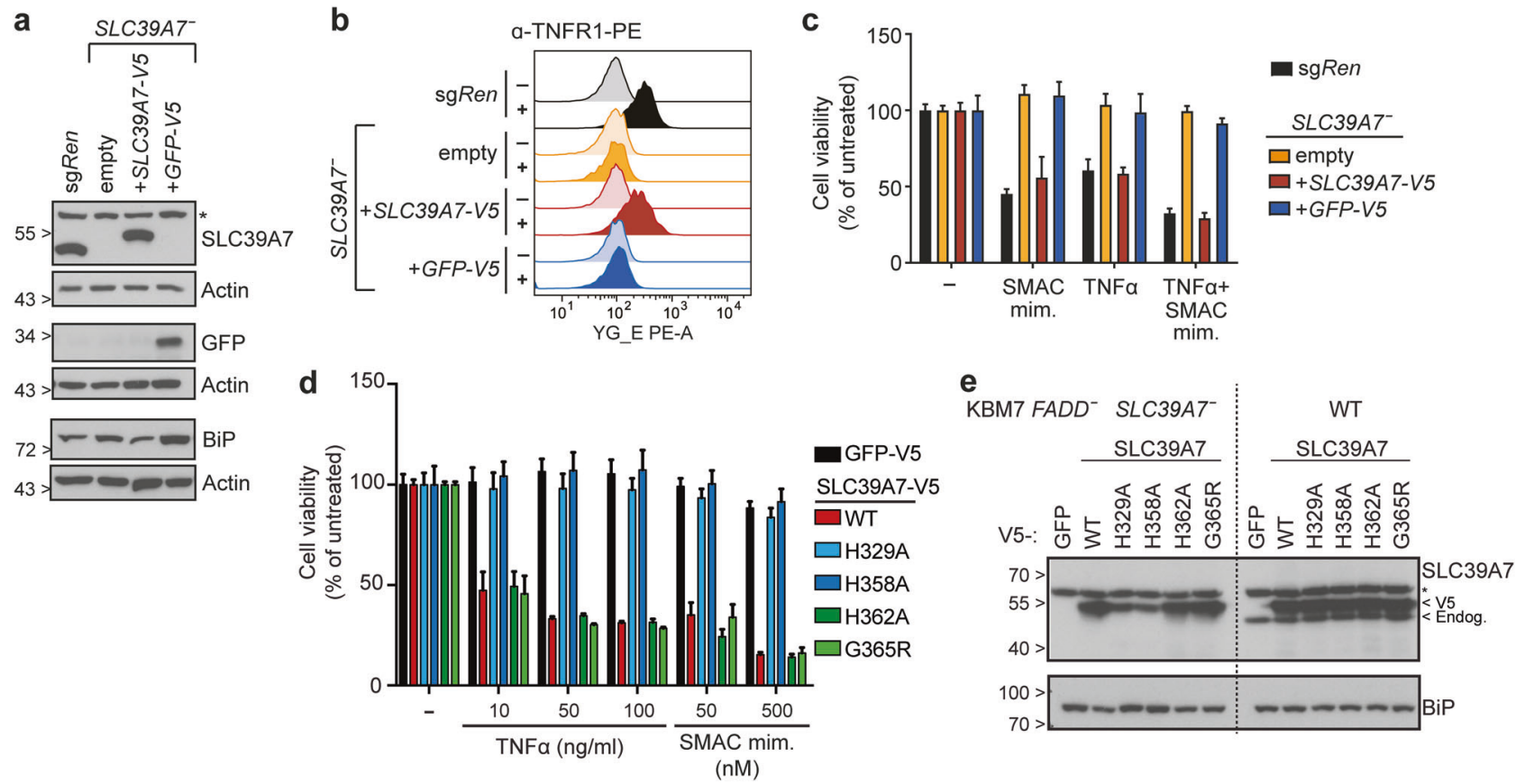

Fig. 5 SLC39A7 transporter function is required to relieve ER stress, restore TNFR1 surface expression and induce cell death. a KBM7 $F A D D^{-}$SLC39A7 cells lentivirally transduced with either V5-tagged SLC39A7 or GFP were lysed and immunoblotted with the indicated antibodies, KBM7 FADD ${ }^{-}$sgRen cells serve as control. Immunoblots shown are representative of two independent experiments, asterisk $(*)$ indicates non-specific band. b Flow cytometry analysis for TNFR1 surface expression in KBM7 FADD ${ }^{-} S L C 39 A 7^{-}$cells reconstituted with either V5-tagged SLC39A7 or GFP. KBM7 FADD sgRen and empty KBM7 FADD $S L C 39 A 7^{-}$cells serve as positive and negative control, respectively. Data shown are representative of two independent experiments. c Cell viability in KBM7 FADD SLC39A7 cells reconstituted with either V5-tagged SLC39A7 or GFP, KBM7

\section{Genome-scale gain-of-function screens identify negative regulators of necroptotic cell death signaling}

Combination of the newly generated KBM7 $F A D D^{-}$cell necroptosis model with recently developed CRISPR/Cas 9 based technologies mediating transcriptional activation of endogenous genes offered the opportunity to identify cellular inhibitors of necroptosis. We thus performed complementary gain-of-function screens employing the CRISPR/Cas9-based SAM approach [22]. KBM7 FADDcells expressing dCas9-VP64 and MS2-p65-HSF1 were transduced with the genome-scale SAM sgRNA lentiviral library and subsequently selected with TNF $\alpha$ or birinapant. NGS sequencing and downstream analysis allowed the identification of sgRNAs specifically enriched upon necroptosis induction, thereby revealing genes conferring resistance to necroptotic cell death when upregulated (Fig. 6a, b, Supplementary Table 6 and 7). Genome-wide screens on birinapant-induced cell death revealed the SMAC mimetic target protein Cellular inhibitor of
$F A D D^{-}$sgRen and empty KBM7 FADD $S L C 39 A 7^{-}$serve as controls. Cells were treated overnight $(16 \mathrm{~h})$ with TNF $\alpha(10 \mathrm{ng} / \mathrm{ml})$, SMAC mimetic $(0.5 \mu \mathrm{M})$, or a combination thereof. Cell viability was assessed using a luminescence-based readout for ATP (CellTiter Glo). Data represent mean value \pm s.d. of two independent experiments performed in triplicates. d Cell viability in KBM7 FADD $S L C 39 A 7^{-}$cells stably reconstituted with GFP or the indicated V5-tagged SLC39A7 constructs. Cells were treated as indicated for $24 \mathrm{~h}$ and cell viability was assessed as in c. Data represent mean value \pm s.d. of two independent experiments performed in triplicates. e KBM7 FADD $S L C 39 A 7^{-}$or KBM7 FADD ${ }^{-}$cells stably reconstituted with the specified constructs were lysed and subjected to immunoblotting with the indicated antibodies. Asterisk $(*)$ indicates non-specific band

apoptosis (cIAP)2 (encoded by BIRC3) as top scoring gene and enriched for sgRNAs targeting cIAP1 (BIRC2), confirming the validity of our approach (Fig. 6b). TNF $\alpha$ treatment similarly revealed BIRC2 among other genes, and, notably, TNIP1 (TNFAIP3 interacting protein 1, ABIN-1) as the top scoring hit (Fig. 6a). Identification of TNIP1 was particularly interesting as this ubiquitin-binding protein affects multiple inflammatory pathways, including TNF receptor and Toll-like receptor signaling [42, 43]. Indeed, TNIP1 was shown to negatively regulate NF-kB and MAPK activation [43, 44] and to inhibit TNF $\alpha$-induced apoptosis [45]. Moreover, single nucleotide polymorphisms (SNPs) in TNIP1 have been associated with several inflammatory diseases [42] and mice deficient for TNIP1 or knock-in for a ubiquitin-binding-defective mutant (TNIP1 [D485N]) develop autoimmunity [46-49]. We first generated KBM7 $F A D D^{-}$cells expressing individual SAMsgRNAs targeting TNIP1, BIRC3 or Renilla as control. Cells overexpressing TNIP1 or cIAP2 (BIRC3) showed increased resistance to $\mathrm{TNF} \alpha$ - or birinapant-induced necroptosis, respectively (Fig. 6c, d, Supplementary 

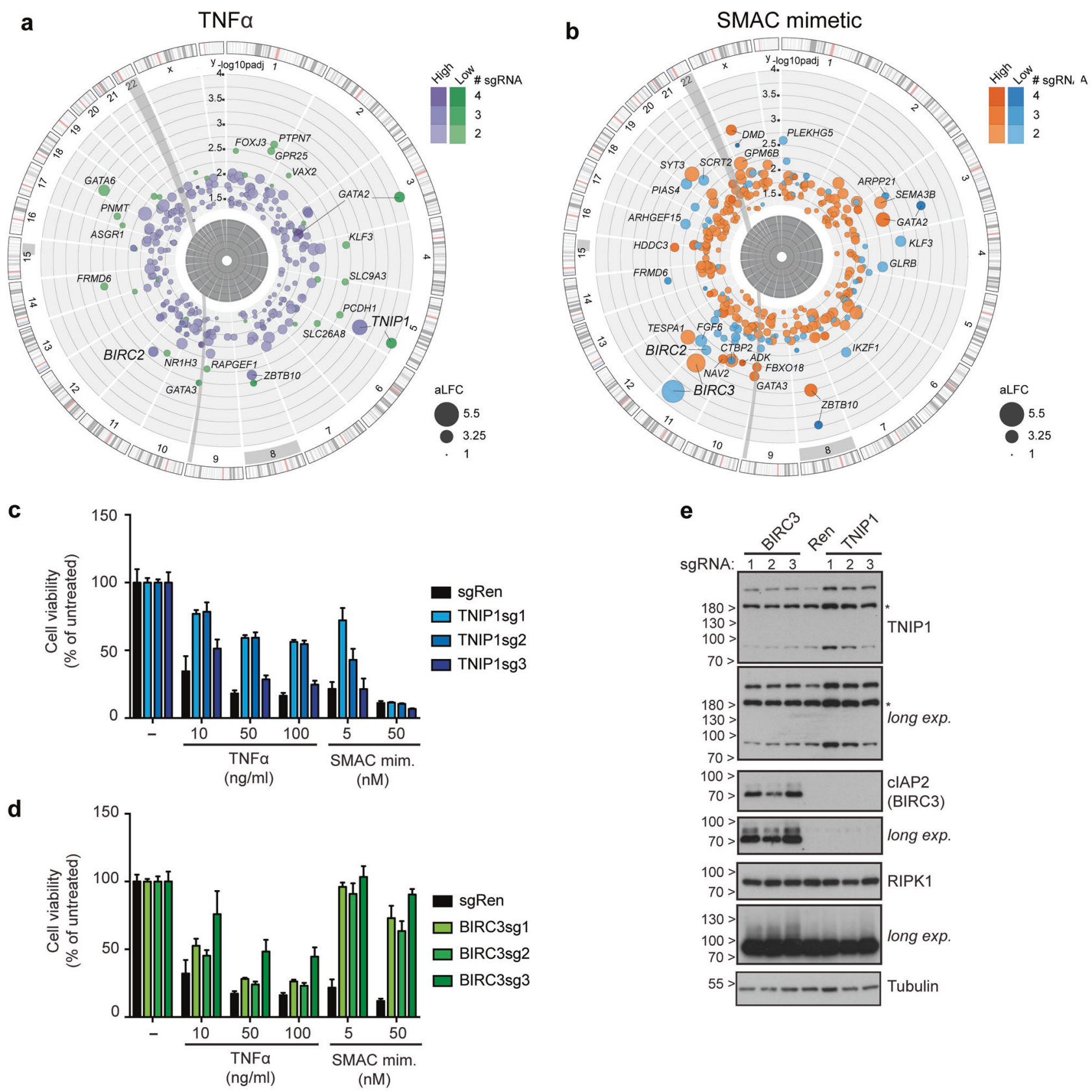

Fig. 6 Genome-scale gene activation screens identify regulators of necroptosis. a, b Circos plots of genome-scale SAM screens in KBM7 $F A D D^{-}$SAM cells with necroptosis induction by TNF $\alpha(a)$ or SMAC mimetic birinapant (b) for $72 \mathrm{~h}$. For each stimulus, screens were performed at low and high concentrations (TNFo: $10 \mathrm{ng} / \mathrm{ml}$ (green) or $100 \mathrm{ng} / \mathrm{ml}$ (purple); birinapant: $0.1 \mu \mathrm{M}$ (blue) or $1 \mu \mathrm{M}$ (orange)). Screen analysis was performed by identifying differentially enriched sgRNAs using DESeq2 and then aggregating sgRNAs to genes using Gene Set Enrichment Analysis. Identified hits are ranked according to the adjusted $p$-value of enrichment $\left(-\log 10\left(p_{\text {adj }}\right)\right.$. Bubble size corresponds to the average $\log 2$ fold-change (aLFC) of enrichment, color

Figure 5a-b). The protective effect of different sgRNAs correlated with the level of overexpression achieved, with the strongest sgRNA for each gene showing partial protection to both stimuli (Fig. 6e). As expected, cIAP2

indicates the number of significantly enriched sgRNAs. Screens were performed in duplicate except the high concentration of birinapant in simplicate. c, d Cell viability in KBM7 $F A D D^{-}$SAM cells transduced with sgRNA targeting TNIP1, BIRC3 or Renilla luciferase (Ren). Cells were treated as indicated for $72 \mathrm{~h}$ and viability was assessed using a luminescence-based readout for ATP (CellTiter Glo). Data represent mean value \pm s.d. of two independent experiments performed in triplicates. e KBM7 FADD ${ }^{-}$SAM cells transduced with the specified sgRNAs were lysed and subjected to immunoblotting with the indicated antibodies. Asterisk $(*)$ indicates non-specific band

upregulation prevented activation of RIPK1 upon birinapant treatment (Supplementary Figure $5 \mathrm{c}$ ). This induced complete degradation of endogenous cIAP1 in both control and cIAP2-overexpressing cells, while only partial depletion of 

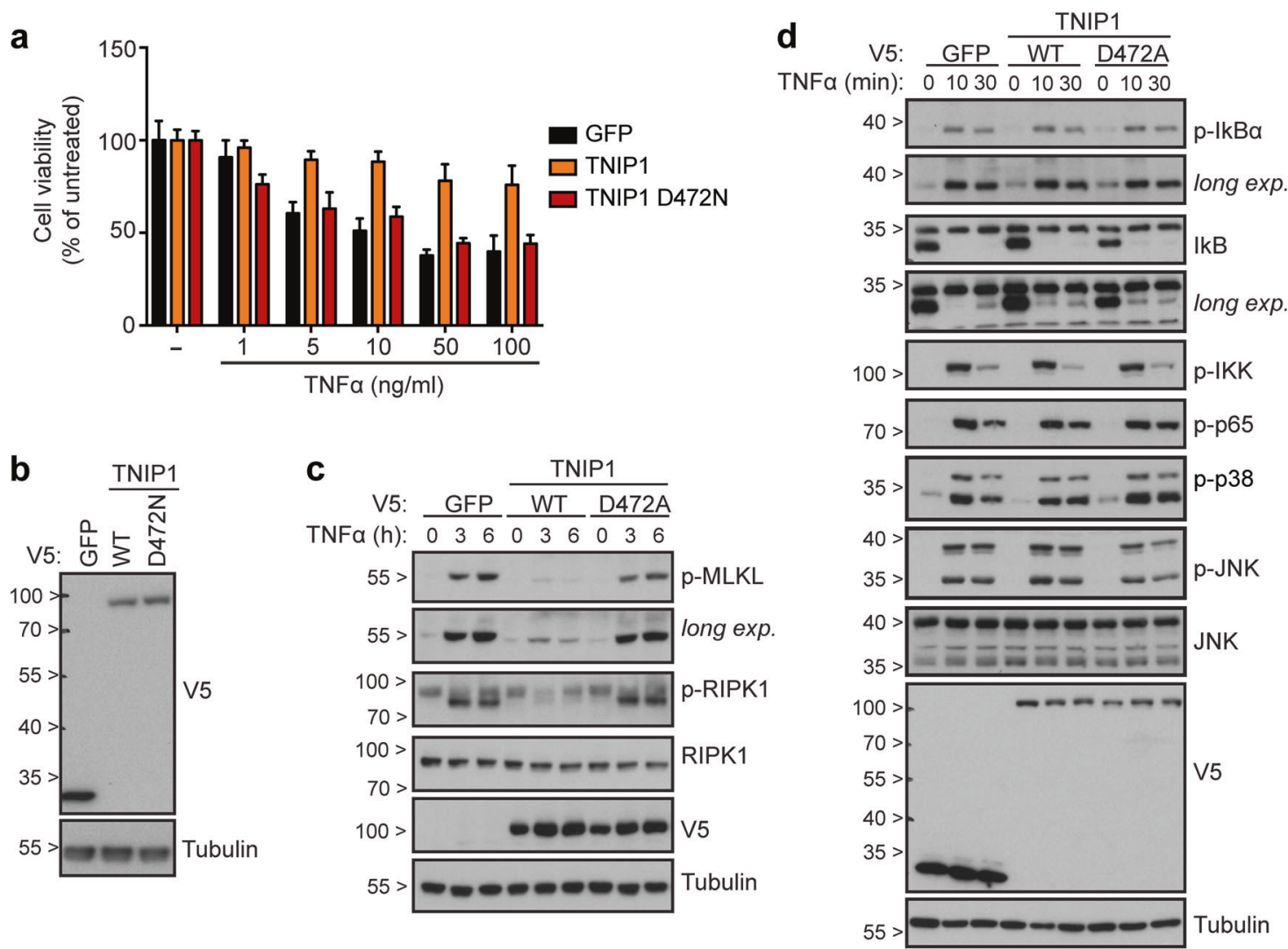

Fig. 7 TNIP1 interferes with TNF $\alpha$-induced necroptosis and RIPK1 activation in a ubiquitin binding-dependent manner. a, b Cell viability (a) and immunoblot (b) of KBM7 FADD cells stably expressing the specified V5-tagged TNIP1 constructs or GFP. In a cells were treated as indicated for $24 \mathrm{~h}$ and viability was assessed using a luminescencebased readout for ATP (CellTiter Glo). Data represent mean value $\pm \mathrm{s}$.

upregulated cIAP2 was observed in the latter, in line with cIAP2 degradation being critically dependent on the presence of cIAP1[50] (Supplementary Figure 5c). Confirming the on-target effects of TNIP1 sgRNAs, cells overexpressing wildtype TNIP1 via ectopic cDNA expression were resistant to TNF $\alpha$-induced necroptosis and showed reduced phosphorylation of RIPK1 and MLKL, indicating that TNIP1 affects the necroptosis pathway upstream of these critical signaling events (Fig. 7a-c). Importantly, expression of a mutant (D472A) disrupting its ubiquitinbinding domain [47] was ineffective, demonstrating that protection requires TNIP1 ubiquitin-binding activity (Fig. 7a-c). In contrast, TNIP1 overexpression did not have any major effect on TNF $\alpha$-induced NF-kB or MAPK pathway activation (Fig. 7d). Similar results were obtained upon sgRNA-mediated TNIP1 overexpression (Supplementary Figure $5 \mathrm{c}-\mathrm{d}$ ).

In summary, these data unveil a role for TNIP1 in controlling $\mathrm{TNF} \alpha$-induced cell death beyond apoptosis. Regulation of necroptosis could therefore contribute to the inflammatory phenotypes observed in TNIP1-deficient and d. of two independent experiments performed in triplicates. (c-d) KBM7 FADD ${ }^{-}$cells stably expressing the specified TNIP1 constructs were stimulated for the time indicated with TNF $\alpha(100 \mathrm{ng} / \mathrm{ml}$ in $\mathbf{c} ; 10$ $\mathrm{ng} / \mathrm{ml}$ in d). Cells were then lysed and subjected to immunoblotting with the indicated antibodies. Data shown are representative of at least two independent experiments

TNIP1[D485N] knock-in mice, and be connected with its association with multiple inflammatory diseases.

\section{Discussion}

Despite its relatively recent discovery, evidence for the relevance of necroptosis in human physiology and pathology has accumulated over the past decade $[4,13,14,51]$. Thus, defining the functional genetic landscape of this fundamental process is expected to expand our understanding of this pathway and reveal potential therapeutic targets. The development of a cellular model efficiently recapitulating necroptosis, while conveniently enabling different types of somatic cell genetic screens, offered the opportunity of attempting a comprehensive survey of human genes involved in the process, both in terms of positive and negative regulation.

We found that deletion of $F A D D$ rendered KBM7 cells sensitive to SMAC mimetic as single agent. SMAC mimetics are considered promising anti-cancer agents and 
birinapant, having shown efficacy as a single agent against some tumors and leukemias [52, 53], is currently being evaluated in clinical trials. SMAC mimetics are known to trigger TNF $\alpha$ production in certain cell lines [54-57] and we observed that phosphorylation of MLKL in KBM7 $F A D D^{-}$cells upon SMAC treatment is delayed compared to TNF $\alpha$ stimulation, in line with the notion of autocrine TNF secretion (Supplementary Figure 1g). Intriguingly, we found that targeting of TNFRSF1B (encoding for TNFR2) in a pooled screen setting rendered cells more resistant to SMAC mimetic-mediated killing, pointing to a cell-intrinsic function. In contrast to TNFR1, TNFR2 can be fully activated only by recognition of membrane-bound, but not soluble TNF $\alpha[58,59]$. Interestingly, activation of TNFR2 has been shown to sensitize macrophages for TNFR1mediated necroptosis [60]. In light of our findings, it will thus be interesting to evaluate whether sensitivity to SMAC mimetic treatment is correlated with TNFR2 expression.

Regarding the other resistance-conferring genes identified, it is tempting to speculate that some of the transcription factors may be directly or indirectly involved in transcriptional regulation of the different pathway components or TNF $\alpha$ itself. Indeed, the basal transcription factor SP1 has been shown to be recruited to the TNF promoter [61, 62] and to mediate RIPK3 expression [63].

The prominent identification across multiple screens of SLC39A7, to date not linked to TNFo signaling, was of particular interest, considering the instrumental role of SLCs in influencing essential physiological processes by regulating metabolic fluxes between the environment and intracellular compartments [64-66]. SLC39A7 controls zinc efflux from the ER to the cytoplasm and its absence has been linked to disturbed zinc homeostasis and ER stress [29-31, 36]. We here experimentally link the resistance to ligand-triggered cell death induction observed in SLC39A7 cells to a trafficking defect of the respective receptors. This is reminiscent of the Notch trafficking abnormalities described for mutants in Catsup, the Drosophila ortholog of SLC39A7 [36]. Whereas we found that basic glycosylation of TNFR1 occurs in SLC39A7 cells, the receptor does not transit to the Golgi apparatus, suggesting that proper folding is not attained. Several of the folding factors in the ER, including protein disulfide isomerases (PDIs) and the chaperones calnexin and calreticulin, rely on zinc as a cofactor and could therefore be affected by SLC39A7 deficiency. Indeed, PDI activity can be inhibited by high zinc concentration [31]. Importantly, reconstitution of SLC39A7 cells with wildtype SLC39A7, but not with mutants predicted to impair its transport activity [40], restored all affected cellular functions indicating that the perturbations induced by SLC39A7 deficiency are reversible, and that substrate-transport is required for its function. Remarkably, the trafficking of specific membrane receptors is differentially affected by SLC39A7 deficiency. We did not identify obvious differences in their posttranslational processing with regard to glycosylation and disulfide bridge formation, and thus the definition of the molecular basis for this divergence remains an intriguing question to be addressed in future studies.

Our data show that SLC39A7 is the prominent SLC affecting TNFR1 and FAS receptor signaling and suggest a largely non-redundant role in ER homeostasis, central processes involved in the pathophysiology of the immune compartment and beyond. It is therefore tempting to speculate that loss-of-function or hypomorphic mutations in SLC39A7 may result in associated genetic diseases. Indeed, defective FASL-induced lymphocyte apoptosis underlies a group of primary immunodeficiencies (PIDs) denoted as autoimmune lymphoproliferative syndrome (ALPS) and our screen with FASL retrieved SLC39A7 amongst genes previously identified in ALPS- and ALPS-like PIDs such as $F A S, F A D D$, and CASP8 (Supplementary Figures 4b) [67]. Mutations in TNFRSF1A, encoding TNFR1, can lead to the autoinflammatory disorder TNFR-associated periodic syndrome (TRAPS) [68], and some variants have been shown to result in abnormal oligomerization and ER retention [69]. Similarly, perturbation of the ER compartment can result in PIDs, as highlighted by the primary antibody deficiency due to plasma cell defects observed in patients with mutations in the Sec61 translocon subunit SEC61A1 [70].

Complementing the loss-of-function approaches, we used CRISPR/Cas9-based gene activation technology to perform genome-scale overexpression screens in analogous settings. Our data highlights a number of candidate genes conferring resistance to necroptotic cell death and revealed a novel role for TNIP1 in inhibiting necroptosis. TNIP1 has been shown to negatively regulate multiple inflammatory pathways through, in particular, its NF-kB inhibitory and anti-apoptotic activities [42, 43, 45]. We demonstrated that TNIP1 overexpression prevented TNF $\alpha$-induced phosphorylation of RIPK1 and of the downstream effector MLKL via a ubiquitin-binding dependent mechanism, while NF-kB and MAPK signaling was largely unaffected. This expands the role of TNIP1 in controlling TNF $\alpha$-induced cell death pathways beyond the proposed role in inhibiting apoptosis by interfering with the recruitment of caspase- 8 by FADD [45]. Considering the association of TNIP1 with multiple inflammatory and autoimmune diseases [42] as well as the TNIP1-dependent phenotypes observed in animal models [46-49, 71], it will be of importance to investigate the relative contribution of TNIP1-dependent necroptosis inhibition we described here. In line with our results, a recent study showed that TNIP1-deficiency sensitizes cells to TNF $\alpha$-induced necroptosis by affecting RIPK1 activation [72]. Upon TNF $\alpha$ treatment, TNIP1 is recruited to the TNF receptor signaling complex by the action of the 
Met1-ubiquitylating complex LUBAC, resulting in the engagement of the deubiquitinating enzyme A20, which targets RIPK1 and limits its activation [72].

In summary, we presented here complementary genomescale loss-of-function and gain-of-function screens in a novel cellular model for necroptosis, and mechanistically validated the role of SLC39A7 and TNIP1 in death receptor trafficking and signaling. Highlighting multiple novel candidate regulators affecting TNF $\alpha$ responses and necroptotic cell death, our work provides the basis for future studies aiming at defining the individual contributions in these central immune processes and exploring the role in associated pathological conditions.

\section{Materials and methods}

\section{Cell lines and reagents}

HEK293T were obtained from ATCC (Manassas, VA, USA). KBM-7 and HAP1 were obtained from T. Brummelkamp. HeLa were provided by M. Hentze, Jurkat E6.1 by W. Ellmeier. KBM7 TNFRI ${ }^{-}$cells were obtained from Haplogen (P00371D06). Cells were cultured in DMEM (Gibco, Grand Island, NY, USA), RPMI (Gibco) or IMDM medium (Gibco) supplemented with 10\% (v/v) FBS (Gibco) and antibiotics $(100 \mathrm{U} / \mathrm{ml}$ penicillin and $100 \mathrm{mg} / \mathrm{ml}$ streptomycin) (Gibco). Cell lines were checked for mycoplasma by PCR or ELISA. The reagents used were as follows: recombinant human TNF- $\alpha$ (300-01A, Peprotech), SMAC mimetic birinapant (S7015, Selleck Chemicals), FasLigand (ALX-522-020-C005, Enzo), recombinant human TRAIL (310-04, Peprotech), z-VAD-FMK (AG-CP3-0002, Adipogen), necrosulfonamide (480073, Merck Millipore), necrostatin-1 (N9037, Sigma-Aldrich), RIPK1 Inhibitor II 7-Cl-ONec-1 (Nec-1s) (504297, Merck Millipore), PNGase F (P0704, NEB), Endo H (P0702, NEB), MG-132 (S2619, Selleckchem), Thapsigargin (P9033, Sigma-Aldrich), Brefeldin A (1231, Tocris), Tunicamycin (T7765, Sigma Aldrich), and doxycycline (D9891, Sigma-Aldrich, St. Louis, MO, USA).

\section{Antibodies}

Antibodies used were phospho-IkBa (Ser32/36) (9246, Cell Signaling), phospho-SAPK/JNK (Thr183/Tyr185) (9251, Cell Signaling), SAPK/JNK (9252, Cell Signaling), IkBa (SC-371, Santa Cruz), phospho-IKK $\alpha / \beta$ (Ser176/180) (2697, Cell Signaling), Phospho-p38 MAPK (Thr180/ Tyr182) (9215, Cell Signaling), Phospho-NF-кB p65 (Ser536) (3033, Cell Signaling), tubulin (ab7291, Abcam), actin (AAN01-A, Cytoskeleton), BiP (610978, BD Biosciences), CHOP (MA1-250, ThermoFisher), SLC39A7
(19429-1-AP, Proteintech), TNFR1 (sc-8436, Santa Cruz), TRAIL-R1/DR4 (42533, Cell Signaling), RIPK1 (610458, BD Bioscience), RIPK3 (12107, Cell Signaling), GFP (sc69779, Santa Cruz), FADD (610399, BD Biosciences), phospho-MLKL (Ser385) (ab187091, Abcam), phosphoRIPK1 (S166) (65746, Cell Signaling), phospho-RIPK3 (S227) (ab209384, abcam), cleaved Caspase-3 (Asp175) (9661, Cell Signaling), V5 (R960-25, Invitrogen or ab9116, abcam), PDIA2 (ab2792, abcam), TNIP1/ABIN-1 (4664, Cell Signaling), cIAP1/BIRC2 (7065, Cell Signaling), cIAP2/BIRC3 (3130, Cell Signaling) and LAMP1 (ab25630, abcam). The secondary antibodies used were goat anti-mouse HRP (115-035-003, Jackson ImmunoResearch), goat anti-rabbit HRP (111-035-003, Jackson ImmunoResearch), Alexa Fluor 680 goat anti-mouse (A21057, Molecular probes) and IRDye 800 donkey antirabbit (611-732-127, Rockland).

\section{Plasmids and cloning}

CRISPR/Cas9-based knockout cell line generation was performed using pX330-U6-Chimeric_BB-CBh-hSpCas9 (Addgene plasmid \#42230), pLentiCRISPRv2 (Addgene plasmid \#52961), or pLentiCas9-BlastR (Addgene plasmid \#52962) and pLentiGuide-PuroR (Addgene plasmid \#52963). To enable color tracing of targeted cells in competition assays, an IRES-GFP or IRES-mCherry fragment was inserted into pLentiGuide-PuroR creating LGPIG (pLentiGuide-PuroR-IRES-GFP) and LGPIC (pLentiGuidePuroR-IRES-mCherry) using standard cloning techniques. CRISPR cloning was performed as described elsewhere [22, 73]. In brief, sgRNAs for KO generation were designed using crispr.mit.edu or CHOPCHOP (chopchop.cbu.uib. no). Oligonucleotides containing BsmBI restriction sitecompatible overhangs were annealed, phosphorylated and ligated into pX330-U6-Chimeric_BB-CBh-hSpCas9, pLentiCRISPRv2, LGPIG or LGPIC using standard cloning techniques and sequence verified using Sanger sequencing. sgRNA targeting Renilla luciferase coding sequence ( $\operatorname{sgRen)}$ was used as a negative control. The sequences of sgRNAs used in this study are listed in Supplementary Table 3. sgRNAs are labeled by target gene name followed by the sequence position of the guide in the corresponding mRNA sequence. Sequences of sgRNAs for SAM-mediated transcriptional activation of TNIP1 and BIRC3 were extracted from hits of the genome-scale library screen and cloned into pLenti-sgRNA(MS2)-zeo backbone (Addgene plasmid \#61427). sgRNA targeting Renilla luciferase coding sequence $(\operatorname{sgRen})$ was used as a negative control and cloned into pLenti-sgRNA(MS2)-zeo-IRES-GFP backbone. SLC39A7 (Clone ID: 3345970, Dharmacon, GE Healthcare) and TNIP1 (HsCD00042052, Harvard Medical School plasmID repository) coding sequence was amplified and 
subcloned into vector pDONR221 using Gateway technology (Invitrogen, Grand Island, NY, USA). Point mutations were introduced by site-directed mutagenesis (InvivoGen). Following sequence verification, cDNAs were transferred into Gateway-compatible expression vectors pLIX403 (Addgene plasmid \#41395) for confocal microscopy experiments, pLX304 (Addgene plasmid \#25890) for SLC39A7 cDNA rescue experiments or pLX302 (Addgene plasmid \#25896) for TNIP1 cDNA overexpression. Retroviral packaging plasmids pGAG-POL and pVSV-G were obtained from $\mathrm{T}$. Brummelkamp, and pADVANTAGE from Promega (E1711). Lentiviral packaging plasmids psPAX2 (plasmid \#12260) and pMD2.G (plasmid \#12259) were from Addgene.

\section{Cell line generation}

HEK293T cells were transiently transfected with pGAGPOL, pVSV-G, pADVANTAGE and retroviral expression vectors, or psPAX2, pMD2.G and lentiviral expression vectors using Polyfect (301105, QIAGEN). After $24 \mathrm{~h}$ the medium was replaced with fresh medium. The viruscontaining supernatant was harvested $48 \mathrm{~h}$ later, filtered $(0.45 \mu \mathrm{m})$, supplemented with $8 \mu \mathrm{g} / \mathrm{ml}$ protamine sulfate (P3369, Sigma-Aldrich) and added to 40-60\% confluent target cells. Suspension cell lines were subjected to spinfection (2000 rpm, $45 \mathrm{~min}$, RT). $24 \mathrm{~h}$ after infection, the medium was replaced with fresh medium supplemented with the respective antibiotics to select for infected cells. Where indicated, target gene expression was induced by adding $2 \mu \mathrm{g} / \mathrm{ml}$ doxycycline. For generation of KBM7 $F A D D^{-}$cells pX330-U6-Chimeric_BB-CBh-hSpCas9 harboring a $F A D D$-targeting sgRNA was electroporated into KBM7 cells using Nucleofector ${ }^{\mathrm{TM}}$ Technology (Lonza). In brief, $2 \times 10^{6}$ cells were spun down, resuspended in $100 \mu \mathrm{l}$ Nucleofection solution (Amaxa Cell Line Nucleofector Kit V, Lonza), and transferred to an electrode cuvette. After addition of $1 \mu \mathrm{g}$ of target vector DNA and electroporation (program Y-005), cells were immediately resuspended in $700 \mu \mathrm{l}$ medium without antibiotics and transferred to a culture vessel with pre-warmed medium. Single cell knockout clones were derived by limiting dilution from KBM7 FADD-Cas9 cells transduced with LGPIG sgSLC39A7_618 or KBM7 FADD-transduced with pLentiCRISPRv2 sgSLC39A7_618.

\section{Haploid genetic screens and deep sequencing analysis}

Haploid genetic screening was conducted as described previously [19, 74]. In brief, gene-trap virus was produced by transient transfection of low passage, subconfluent HEK293T cells with the gene-trap plasmid and packaging plasmids pGAG-POL, pVSV-G and pADVANTAGE using Lipofectamine 2000 (Thermo Fisher Scientific). Every $24 \mathrm{~h}$ for three consecutive times the virus-containing supernatant was collected and replaced with fresh medium. Virus was concentrated via ultracentrifugation and used to mutagenize $1 \times 10^{8} \mathrm{KBM} 7$ cells by spinfection. Mutagenized cells were then expanded and directly used for genetic screens, while $1 \times 10^{8}$ gene-trapped cells were harvested as unselected control population. For screens, a total number of $1 \times 10^{8}$ gene-trapped cells/screen was selected with the indicated stimuli (TRAIL $100 \mathrm{ng} / \mathrm{ml}$, FASL $250 \mathrm{ng} / \mathrm{ml}$ ) in 96-well plates $\left(1 \times 10^{5}\right.$ cells/well in $\left.100 \mu \mathrm{l}\right)$. After 12 days, resistant clones were pooled, cleaned up using Lonza lymphocyte separation buffer (17-829E), expanded to a total number of $3-6 \times 10^{7}$ cells, and genomic DNA was isolated.

To map insertions by next generation sequencing, retroviral insertion sites in necroptosis screen samples and unselected reference pools were recovered via linear amplification-mediated (LAM)-PCR, and FASL and TRAIL screen samples were digested with NlaIII and MseI and circularized, followed by inverse PCR. Samples were loaded onto an Illumina HiSeq 2000 machine using custom sequencing primer [18] and sequenced 50 base pair singleend. The obtained sequences in the FASTQ data file were mapped to the human reference genome hg19 (UCSC hg19 build) using bowtie2 (version 2.2.4) with default parameters. Sequencing reads with multiple alignment as well as reads with low mapping quality $(\mathrm{MAPQ}<20$ ) were discarded. Duplicate reads were marked and discarded with Picard (version 1.118). Insertions 1 or 2 base pairs away from each other were removed to avoid inclusion of insertions due to mapping errors. Each uniquely mapped read corresponds to an insertion site and was annotated with gene build GRCh37.p13 (ENSEMBL 75-release February 2014) using bedtools (version 2.10.1) and custom scripts. Only insertions that are predicted to be disruptive to gene function were further tested for significant enrichment. The significance of enrichment of insertions in a given gene was calculated by comparing the number of insertions in the selected populations with the unselected control data set by applying a one-sided Fisher's exact test. P-values were adjusted for false discovery rate (FDR) using BenjaminiHochberg procedure (5\% threshold was considered significantly enriched). Screen results were visualized using Circos (version 0.66) software, summary bubble plots and gene-trap insertion plots were generated via custom scripts using R statistical environment.

\section{CRISPR/Cas9-based genetic screens}

The SLC KO CRISPR/Cas 9 library used is described in detail in [75] and Girardi et al. (in preparation). Briefly, a CRISPR/Cas9 library targeting 388 SLC genes with six 
sgRNAs per gene, together with a set of 120 sgRNAs targeting 20 genes essential in KBM7 and HAP1 cells [35], and a set of 120 non-targeting sgRNAs was cloned by Gibson cloning in the pLentiCRISPRv2 lentiviral vector. Viral particles were generated by transient transfection of low passage, subconfluent HEK293T cells with the SLCtargeting library and packaging plasmids pGAG-POL and pVSV-G using PolyFect (Qiagen). After $24 \mathrm{~h}$ the media was changed to fresh IMDM media supplemented with $10 \%$ FCS and antibiotics. The viral supernatant was collected after $48 \mathrm{~h}$, filtered and stored at $-80^{\circ} \mathrm{C}$ until further use. The supernatant dilution necessary to infect KBM7 FADD cells at a MOI (multiplicity of infection) of $0.2-0.3$ was determined by puromycin survival after transduction as described in the ref. [76]. KBM7 FADD cells were infected in duplicates with the SLC KO library at high coverage $(1000 \times)$ and after selection for 7 days with puromycin $(0.5$ $\mu \mathrm{g} / \mathrm{ml})$ an initial sample was collected to control for library composition. Cells were then treated with a set of stimuli (KBM7 FADD: TNF $\alpha 50 \mathrm{ng} / \mathrm{ml}, 12$ days; TNF $\alpha 10 \mathrm{ng} / \mathrm{ml}$, 12 days; SMAC mimetic $5 \mu \mathrm{M}, 12$ days; SMAC mimetic 1 $\mu \mathrm{M}, 12$ days; KBM7 wildtype: TRAIL $100 \mathrm{ng} / \mathrm{ml}, 14$ days; FASL $100 \mathrm{ng} / \mathrm{ml}, 10$ days; HAP1 wildtype: TRAIL $100 \mathrm{ng} /$ $\mathrm{ml}, 9$ days; FASL $100 \mathrm{ng} / \mathrm{ml}, 7$ days) and cell samples collected from both treated and control samples. Genomic DNA was extracted using the DNAeasy kit (QIAGEN) and the cassette containing the sgRNA sequence amplified with two rounds of PCRs following the procedure described in the ref. [76].

The amplified samples were sequenced on a HiSeq3000/ 4000 (Illumina), followed by processing with a custom analysis pipeline (see next section).

Genome-scale SAM screen was performed following an analogous procedure as previously described [22]. In brief, KBM7 FADD ${ }^{-}$cells were transduced first with lenti MS2P65-HSF1 (Addgene plasmid \#61426; selection Hygromycin $800 \mu \mathrm{g} / \mathrm{ml}$ ) and subsequently with lenti dCAS-VP64 (Addgene plasmid \#61425, selection Blasticydin $20 \mu \mathrm{g} / \mathrm{ml}$ ) to generate KBM7 FADD $D^{-} \mathrm{SAM}$ cells. The Human CRISPR/Cas9 Synergistic Activation Mediator (SAM) pooled sgRNA library (Addgene \#1000000057) was amplified and packaged into lentivirus following the same procedure described above for the SLC KO CRISPR/Cas9 library. After viral titration, $2 \times 10^{8}$ KBM7 $F A D D^{-}$ CRISPR-SAM cells were infected at $0.3 \mathrm{MOI}$ and selected $48 \mathrm{~h}$ later with zeomycin $(150 \mu \mathrm{g} / \mathrm{ml})$ for 8 days. $5 \times 10^{6}$ cells were either harvested (time 0 control), treated in duplicate with two doses of TNF $\alpha(10$ or $100 \mathrm{ng} / \mathrm{ml})$ or SMAC mimetic $(0.1$ or $1 \mu \mathrm{M})$ or left untreated (untreated control). After $72 \mathrm{~h}$ cells were washed, expanded for 7 days before isolation of live cells by Lymphoprep (Stemcell technologies). Genomic DNA was extracted using the DNAeasy Blood \& Tissue kit (QIAGEN) and the cassette containing the sgRNA sequence amplified using NEBnext High Fidelity 2X Master Mix (New England Biolabs) in 36 single-step PCR reactions of 23 cycles per sample with primers introducing sequencing adapters and barcodes (described in the ref. [22]). PCR products were pooled, purified and gel extracted. The amplified samples were then quantified, pooled (maximum of eight samples per sequencing lane) and sequenced on a HiSeq3000/4000 (Illumina), followed by processing with a custom analysis pipeline (see next section).

\section{Analysis of CRISPR screens}

Sequences of sgRNAs were extracted from RNA-Seq reads, matched against the original sgRNA library index and counted using an in-house Python script. To compensate for the noise and off target action of sgRNAs inherent to CRISPR screening approaches, we used a two-step differential abundance analysis. In a first step, differential abundance of sgRNAs was estimated with DESeq2 [77] using a two-factor design that accounts for both time and treatment variables. Subsequently, significantly enriched sgRNAs (adjusted $p$-value $\leq 0.05$ ) were sorted by $\log 2$-fold change and aggregated to genes using Gene Set Enrichment Analysis [78], only considering genes with at least two significantly enriched sgRNAs.

\section{T7 Endonuclease assay}

To determine $F A D D$ targeting efficiency, $1-3 \times 10^{6}$ cells were spun down, washed in 1x PBS and genomic DNA isolated using the DNeasy Blood \& Tissue Kit (QIAGEN) according to the manufacturer's instructions. The CRISPR/ Cas 9 targeting site-containing locus was PCR-amplified (fw primer: GAGCTGACCGAGCTCAAGTTCCTAT; rv primer: CAAATCAAACCCGGCAAAGG; product size: 342 bp). The PCR products were denatured at $95^{\circ} \mathrm{C}$ for $2 \mathrm{~min}$ and re-annealed by ramping from $95-85^{\circ} \mathrm{C}$ at $-2{ }^{\circ} \mathrm{C} / \mathrm{s}$ and from 85 to $25^{\circ} \mathrm{C}$ at $-0.1{ }^{\circ} \mathrm{C} / \mathrm{s}$. The annealed PCR products were digested with T7 Endonuclease I (NEB, M0302) for $20 \mathrm{~min}$ at $37^{\circ} \mathrm{C}$, control digests without T7 Endonuclease I served as negative control. The fragmented PCR products were analysed by agarose gel electrophoresis.

\section{Immunoblotting}

Whole cell extracts were prepared using Nonidet-40 lysis buffer $(50 \mathrm{mM}$ HEPES pH $7.4,250 \mathrm{mM} \mathrm{NaCl}, 5 \mathrm{mM}$ EDTA, $1 \% \mathrm{NP}-40,10 \mathrm{mM} \mathrm{NaF}$ and $1 \mathrm{mM} \mathrm{Na3VO} 4$ or Halt phosphatase inhibitor cocktail (ThermoScientific), one tablet of EDTA-free protease inhibitor (Roche) per $50 \mathrm{ml}$ ) for $10 \mathrm{~min}$ on ice. Lysates were cleared by centrifugation (13000 rpm, $10 \mathrm{~min}, 4^{\circ} \mathrm{C}$ ). The proteins were quantified 
with BCA (Pierce, Grand Island, NY, USA) or Bradford assay (Bio-Rad, Hercules, CA, USA). Cell lysates were resolved by SDS-PAGE and transferred to nitrocellulose membranes Protran BA 85 (GE Healthcare, Little Chalfont, UK). The membranes were immunoblotted with the indicated antibodies. Bound antibodies were visualized with horseradish peroxidase-conjugated secondary antibodies using the ECL Western blotting system (Thermo Scientific, Waltham, MA, USA) or Odyssey Infrared Imager (LI-COR, Lincoln, NE, USA).

\section{Cell viability assays}

Cells were seeded in 96-well plates at the appropriate cell density. For cell death induction, cells were incubated with the indicated compounds as stated or overnight (14-16 h). Cell viability was determined using CellTiter Glo Luminescent Cell Viability Assay (Promega, Fitchburg, WI, USA) according to the instructions provided by the manufacturer. Luminescence was recorded with a SpectraMax M5Multimode plate reader (Molecular Devices, Sunnyvale, CA, USA). Data were normalized to values of untreated controls.

\section{Flow cytometry}

For flow cytometric analyses, cells were blocked with PBS, $10 \%$ FBS for $10 \mathrm{~min}$ on ice and subsequently stained with PE-labeled mouse anti-human TNFR1 (R\&D Systems, FAB225P), PE-Cy ${ }^{\mathrm{TM}}$ 7-labeled mouse anti-human CD95 (BD Biosciences, 561633), PE-labeled mouse anti-human TRAIL-R1/DR4 (eBioscience, 12-6644-41), PE-labeled mouse anti-human TRAIL-R2/DR5 (eBioscience, 129908-41), APC-labeled mouse anti-human C3AR (BioLegend, 345805), PE-labeled mouse anti-human C5L2 (BioLegend, 342403), FITC-labeled mouse anti-human CD11a (eBioscience, 11-0119-41), PE-labeled mouse antihuman CD123 (eBioscience, 12-1239-42), PE-Cyanine5labeled mouse anti-human CD14 (eBioscience, 15-014941), APC-labeled mouse anti-human CD31 (eBioscience, 17-0319-42), $\quad \mathrm{Cy}^{\mathrm{TM}}$ 7-labeled mouse anti-human CD34 (BD Pharmingen, 560710), PerCP-Cyanine5.5-labeled mouse anti-human CD4 (eBioscience, 45-0049120), PerCP-Cyanine5.5-labeled mouse anti-human CD44 (BD Pharmingen, 560531), PerCP-Cyanine5.5-labeled mouse anti-human CD45 (eBioscience,45-0459-42), PE-labeled mouse anti-human CD54 (BD Pharmingen, 560971), PE-labeled mouse anti-human CD55 (BD Pharmingen, 561901), or PerCP-Cyanine5.5-labeled mouse anti-human HLA-ABC (BD Pharmingen, 555554). All stains were performed in FACS buffer (PBS, 10\% FBS) for $30 \mathrm{~min}$ in the dark at either RT or $4{ }^{\circ} \mathrm{C}$, followed by two washing steps. Samples were analyzed on an LSR Fortessa
(BD Biosciences) or FACSCalibur (BD Biosciences). Dead cells were excluded by forward- and side-scatter, and data analysis was performed using FlowJo software version 7.6.3 (Tree Star Inc., Ashland, OR, USA).

\section{Flow cytometry-based multi-color competition assay (MCA)}

To analyze the long-term cellular response upon treatment with different cell death inducers in CRISPR/Cas9-based knockout cell competition experiments, cell populations were marked with a fluorescent reporter coupled to individual sgRNAs, with sgRNA targeting Renilla luciferase (sgRen) serving as control. $\mathrm{sgRen}$-mCherry ${ }^{+}$reporter cells (LGPIC lentiviral sgRNA vector) were mixed with $\mathrm{sgRen-}$ $\mathrm{GFP}^{+}$control or target gene sgRNA-GFP ${ }^{+}$cells (LGPIG lentiviral vector) in a 1:1 ratio. The mixed cell populations were treated with the cell death inducers indicated or left untreated as reference. After 14 days, the respective percentage of mCherry ${ }^{+}$and $\mathrm{GFP}^{+}$was determined by flow cytometry, gating on viable cells (FSC/SSC).

\section{Confocal microscopy}

HeLa cells inducibly expressing V5-tagged SLC39A7 were plated on glass coverslips and expression induced by addition of $2 \mu \mathrm{g} / \mathrm{ml}$ doxycycline. After $24 \mathrm{~h}$, cells were washed with 1x PBS, fixed (PBS, $4 \%$ formaldehyde) and permeabilized (PBS, $0.3 \%$ saposin, 10\% FBS). Subsequently, coverslips were incubated with anti-V5 (Invitrogen, R960-25) and anti-Calreticulin (abcam, ab2907) for $1 \mathrm{~h}$ at RT in PBS, $0.3 \%$ saposin, $10 \%$ FBS. After three washes, coverslips were stained with goat anti-mouse AlexaFluor 568 (Invitrogen, A-11004) and anti-rabbit AlexaFluor488 (Invitrogen, A-11008) antibodies followed by DAPI staining. Coverslips were washed three times and mounted on glass slides using ProLong Gold (Invitrogen, P36934). Images were taken with a Zeiss Laser Scanning Microscope (LSM) 700. Images were taken with a $63 \times$ oil immersion objective (na 1.4) and exported from lsm to tiff files.

\section{Triton X-114 phase separation for enrichment of membrane proteins}

Triton X-114 phase separation was performed as described previously [79]. Briefly, $60-75 \times 10^{6}$ cells were harvested and washed once with PBS. Cells were then resuspended in $500 \mu \mathrm{l}$ PBS and $100 \mu \mathrm{l} 6 \%$ pre-condensed Triton X-114, mixed by inversion and incubated for $15 \mathrm{~min}$ on ice. After centrifugation for $1 \mathrm{~min}$ at $13,000 \mathrm{rpm}$, the supernatants were transferred to new tubes and incubated for $5 \mathrm{~min}$ at $37^{\circ} \mathrm{C}$ to induce phase separation. The upper aqueous phase was transferred to a new tube. To wash, $500 \mu$ PBS were 
added to the detergent phase and $100 \mu \mathrm{l} 6 \%$ Triton X-114 to the aqueous phase, followed by incubation for $5 \mathrm{~min}$ on ice and $5 \mathrm{~min}$ at $37^{\circ} \mathrm{C}$. Samples were centrifuged as before and the initial phases were kept for further processing. Proteins in the detergent phase were precipitated by adding $450 \mu \mathrm{l}$ PBS, $500 \mu \mathrm{l}$ methanol and $125 \mu \mathrm{l}$ chloroform followed by vortexing. After centrifugation for $4 \mathrm{~min}$ at $13,000 \mathrm{rpm}, 750 \mu \mathrm{l}$ of the supernatant were removed, $400 \mu \mathrm{l}$ methanol added and mixed by pipetting. Samples were centrifuged for $1 \mathrm{~min}$ at $13,000 \mathrm{rpm}$, the supernatant removed and the pellets dried at RT. Subsequently, the proteins were resuspended by sonication in $100 \mu$ l SDSPAGE sample buffer without glycerol and bromophenol blue. Samples were then either subjected to immunoblot analysis or further prepared for mass spectrometric analysis via the FASP (filter aided sample preparation) method as described previously [80].

\section{Reversed-phase liquid chromatography mass spectrometry (LCMS)}

Mass spectrometry was performed on a hybrid linear trap quadrupole (LTQ) Orbitrap Velos mass spectrometer (ThermoFisher Scientific, Waltham, MA) using the Xcalibur version 2.1.0 coupled to an Agilent 1200 HPLC nanoflow system (dual pump system with one pre-column and one analytical column) (Agilent Biotechnologies, Palo Alto, CA) via a nanoelectrospray ion source using liquid junction (Proxeon, Odense, Denmark). Solvents for LCMS separation of the digested samples were as follows: solvent A consisted of $0.4 \%$ formic acid in water and solvent B consisted of $0.4 \%$ formic acid in $70 \%$ methanol and $20 \%$ isopropanol. From a thermostatic microautosampler, $8 \mu \mathrm{l}$ of the tryptic peptide mixture were automatically loaded onto a trap column (Zorbax 300SB-C18 $5 \mu \mathrm{m}, 5 \times 0.3 \mathrm{~mm}$, Agilent Biotechnologies) with a binary pump at a flow rate of $45 \mu \mathrm{l} /$ min. $0.1 \%$ TFA was used for loading and washing the precolumn. After washing, the peptides were eluted by back-flushing onto a $16 \mathrm{~cm}$ fused silica analytical column with an inner diameter of $50 \mu \mathrm{m}$ packed with $\mathrm{C} 18$ reversed phase material (ReproSil-Pur 120 C18-AQ, $3 \mu \mathrm{m}$, Dr. Maisch, Ammerbuch-Entringen, Germany). The peptides were eluted from the analytical column with a 27 min gradient ranging from 3 to $30 \%$ solvent $\mathrm{B}$, followed by a 25 min gradient from 30 to $70 \%$ solvent B and, finally, a 7 min gradient from 70 to $100 \%$ solvent $\mathrm{B}$ at a constant flow rate of $100 \mathrm{n} ; / \mathrm{min}$. The analyses were performed in a datadependent acquisition mode using a top 10 collisioninduced dissociation (CID) method. Dynamic exclusion for selected ions was $60 \mathrm{~s}$. A single lock mass at $\mathrm{m} / \mathrm{z}$ 445.120024 was employed. The maximal ion accumulation time for MS in the orbitrap and MS2 in the linear trap was 500 and $50 \mathrm{~ms}$, respectively. Automatic gain control (AGC) was used to prevent overfilling of the ion traps. For MS and MS2, AGC was set to $10^{6}$ and 5000 ions, respectively. Peptides were detected in MS mode at a resolution of 60,000 (at $\mathrm{m} / \mathrm{z}$ 400). The threshold for switching from MS to MS2 was 2000 counts. All samples were analysed as technical, back-to-back replicates.

\section{Mass spectrometry data processing and analysis}

The acquired raw MS data files were processed with msconvert (ProteoWizard Library v2.1.2708) and converted into Mascot generic format (mgf) files. The resultant peak lists were searched against either the human or mouse SwissProt database v2014.07 (40,984 and 24,862 sequences, respectively, including isoforms obtained from varsplic. pl and appended with known contaminants) with the search engines Mascot (v2.3.02, MatrixScience, London, U.K.) and Phenyx (v2.5.14, GeneBio, Geneva, Switzerland). Submission to the search engines was via a Perl script that performs an initial search with relatively broad mass tolerances (Mascot only) on both the precursor and fragment ions ( $\pm 10 \mathrm{ppm}$ and $\pm 0.6 \mathrm{Da}$, respectively). High-confidence peptide identifications were used to recalibrate all precursor and fragment ion masses prior to a second search with narrower mass tolerances $( \pm 4 \mathrm{ppm}$ and $\pm 0.3 \mathrm{Da}$, respectively). One missed tryptic cleavage site was allowed. Carbamidomethyl cysteine and oxidised methionine were set as fixed and variable modifications, respectively. To validate the proteins, Mascot and Phenyx output files were processed by internally-developed parsers. Proteins with $\geq 2$ unique peptides above a score $\mathrm{T} 1$ or with a single peptide above a score $\mathrm{T} 2$ were selected as unambiguous identifications. Additional peptides for these validated proteins with score $>\mathrm{T} 3$ were also accepted. For Mascot and Phenyx, $\mathrm{T} 1, \mathrm{~T} 2$, and $\mathrm{T} 3$ peptide scores were equal to $14,28,10$ and $4.2,4.7,3.5$, respectively $\left(p\right.$-value $<10^{-3}$ ). The validated proteins retrieved by the two algorithms were merged, any spectral conflicts discarded and grouped according to shared peptides. A false discovery rate of $<1 \%$ for protein identifications and $<1 \%$ for peptides (including the ones exported with lower scores) was determined by applying the same procedure against a database of reversed protein sequences. Commonly-known contaminants including trypsin and keratin were removed. In each condition, four spectral counts were available for the median (two biological replicates and two technical for each). Average spectral counts of the replicates were used to determine $\log 2$ fold changes between KBM7 FADD- SpCas9 SLC39A7 vs. sgRen control. $p$-values were calculated by a two-sided Welsch Two Sample t-test. Annotations for the GO term "GO:0034976 response to endoplasmic reticulum stress" were obtained from QuickGO (www.ebi.ac.uk/QuickGO). GO term enrichment was computed with R's topGO 
package for biological process ontology, using the default algorithm ("weight01") and the annotation file from geneontology.org as of October 2016. The study set consisted in all proteins identified with a $\log 2$ fold change $\geq 1$ and a $p$-value $<0.05$ ( 65 proteins). All reviewed human proteins in UniProtKB/Swiss-Prot were used as background population $(20,348$ proteins). $p$-values were corrected for multiple testing using Benjamini-Hochberg's procedure (False Discovery Rate). Gene Set Enrichment Analysis was performed using the GSEA software from the Broad Institute with default parameters. The gene list consisted in all proteins identified below a $p$-value of 0.05 (354 proteins), ranked by $\log 2$ fold change. The analysis was run against the Hallmarks gene set. Supplementary Table 2 gives results for LC-MSMS analysis, GO term enrichment, and GSEA.

\section{Experimental design, data plotting and statistical rational}

Proteomics experiments were performed as biological replicates $(n=2)$ and analyzed by LC-MSMS as technical duplicates. CRISPR/Cas 9 library screens were performed in duplicate $(n=2)$. Flow cytometry-based MCA data are shown as mean value \pm s.d. of at least two independent experiments $(n \geq 2)$ performed in duplicates. Data calculations were performed using Microsoft Excel (Microsoft, Redmond, WA, USA), data plotting and statistical analysis was done using GraphPad Prism 6 (GraphPad Software) if not otherwise stated. A normal distribution of data was assumed and appropriate tests were applied. Visualization of data was performed using $\mathrm{R}$ statistical environment.

Acknowledgements We thank all members of the Superti-Furga laboratory for discussions and feedback, Georg E. Winter for support with the haploid genetic screens, Hawwa O. Oshafu for technical help, the Proteomics Facility for the proteomics analyses and the Biomedical Sequencing facility for the NGS sequencing. This work was supported by the Austrian Academy of Sciences (G.S.-F., A.F., K. H.), the Ludwig Boltzmann Society (K.B.), the European Research Council (ERC AdG 695214 GameofGates S.S., M.B., M.R.), EMBO long-term fellowship (ALTF 1346-2011 M.R.), Marie SklodowskaCurie fellowship (IEF-301663 M.R.; IF-661491 E.G.), Austrian Science Fund Lise Meitner Program Fellowship (FWF M1809 K.L.W.), Austrian Science Fund Grants (FWF F47-B20 J.W.B., F.S.; FWF I2192 A.C.-R.; FWF P29250 E.G., J.K.) and Boehringer Ingelheim (Research Collaboration Agreement BI-CeMM 238114 L.X.H. and M. R.). Plasmids obtained through Addgene were a gift from Feng Zhang, David Root and Didier Trono.

Author contributions A.F., M.R., K.L.W., K.B. and G.S-F. designed research; A.F., M.R., K.L.W., E.G., S.S., M.B. and J.K. performed research; E.G. generated reagents and provided scientific insight; A.CR. and F.S. analyzed genetic screening data; J.W.B., L.X.H. and K.H. provided reagents and gave experimental advice; A.F., M.R., K.L.W. and G.S-F. analyzed and interpreted the data; A.F., M.R. and G.S-F. wrote the paper.

\section{Compliance with ethical standards}

Conflict of interest The Superti-Furga laboratory at CeMM has an active research collaboration with Boehringer Ingelheim (Research Collaboration Agreement BICeMM 238114). The remaining authors declare that they have no conflict of interests.

Open Access This article is licensed under a Creative Commons Attribution 4.0 International License, which permits use, sharing, adaptation, distribution and reproduction in any medium or format, as long as you give appropriate credit to the original author(s) and the source, provide a link to the Creative Commons license, and indicate if changes were made. The images or other third party material in this article are included in the article's Creative Commons license, unless indicated otherwise in a credit line to the material. If material is not included in the article's Creative Commons license and your intended use is not permitted by statutory regulation or exceeds the permitted use, you will need to obtain permission directly from the copyright holder. To view a copy of this license, visit http://creativecommons. org/licenses/by/4.0/.

\section{References}

1. Pasparakis M, Vandenabeele P. Necroptosis and its role in inflammation. Nature. 2015;517:311-20.

2. Chan FK, Luz NF, Moriwaki K. Programmed necrosis in the cross talk of cell death and inflammation. Annu Rev Immunol. 2015;33: 79-106.

3. Orozco S, Oberst A. RIPK3 in cell death and inflammation: the good, the bad, and the ugly. Immunol Rev. 2017;277:102-12.

4. Grootjans S, Vanden Berghe T, Vandenabeele P. Initiation and execution mechanisms of necroptosis: an overview. Cell Death Differ. 2017;24:1184-95.

5. Cho YS, Challa S, Moquin D, Genga R, Ray TD, Guildford M, et al. Phosphorylation-driven assembly of the RIP1-RIP3 complex regulates programmed necrosis and virus-induced inflammation. Cell. 2009;137:1112-23.

6. He S, Wang L, Miao L, Wang T, Du F, Zhao L, et al. Receptor interacting protein kinase-3 determines cellular necrotic response to TNF-alpha. Cell. 2009;137:1100-11.

7. Zhang DW, Shao J, Lin J, Zhang N, Lu BJ, Lin SC, et al. RIP3, an energy metabolism regulator that switches TNF-induced cell death from apoptosis to necrosis. Science. 2009;325:332-6.

8. Sun L, Wang H, Wang Z, He S, Chen S, Liao D, et al. Mixed lineage kinase domain-like protein mediates necrosis signaling downstream of RIP3 kinase. Cell. 2012;148:213-27.

9. Zhao J, Jitkaew S, Cai Z, Choksi S, Li Q, Luo J, et al. Mixed lineage kinase domain-like is a key receptor interacting protein 3 downstream component of TNF-induced necrosis. Proc Natl Acad Sci USA. 2012;109:5322-7.

10. Degterev A, Hitomi J, Germscheid M, Ch'en IL, Korkina O, Teng $\mathrm{X}$, et al. Identification of RIP1 kinase as a specific cellular target of necrostatins. Nat Chem Biol. 2008;4:313-21.

11. Silke J, Rickard JA, Gerlic M. The diverse role of RIP kinases in necroptosis and inflammation. Nat Immunol. 2015;16:689-97.

12. Linkermann A, Green DR. Necroptosis. N Engl J Med. 2014;370: 455-65.

13. Galluzzi L, Kepp O, Chan FK, Kroemer G. Necroptosis: mechanisms and relevance to disease. Annu Rev Pathol. 2017;12: 103-30.

14. Degterev A, Huang Z, Boyce M, Li Y, Jagtap P, Mizushima N, et al. Chemical inhibitor of nonapoptotic cell death with therapeutic potential for ischemic brain injury. Nat Chem Biol. 2005; 1:112-9. 
15. Lin J, Li H, Yang M, Ren J, Huang Z, Han F, et al. A role of RIP3-mediated macrophage necrosis in atherosclerosis development. Cell Rep. 2013;3:200-10.

16. Linkermann A, Brasen JH, Darding M, Jin MK, Sanz AB, Heller $\mathrm{JO}$, et al. Two independent pathways of regulated necrosis mediate ischemia-reperfusion injury. Proc Natl Acad Sci USA. 2013;110:12024-9.

17. Upton JW, Shubina M, Balachandran S. RIPK3-driven cell death during virus infections. Immunol Rev. 2017;277:90-101.

18. Carette JE, Guimaraes CP, Varadarajan M, Park AS, Wuethrich I, Godarova A, et al. Haploid genetic screens in human cells identify host factors used by pathogens. Science. 2009;326:1231-5.

19. Carette JE, Guimaraes CP, Wuethrich I, Blomen VA, Varadarajan $\mathrm{M}$, Sun $\mathrm{C}$, et al. Global gene disruption in human cells to assign genes to phenotypes by deep sequencing. Nat Biotechnol. 2011; 29:542-6.

20. Gilbert LA, Horlbeck MA, Adamson B, Villalta JE, Chen Y, Whitehead EH, et al. Genome-scale CRISPR-mediated control of gene repression and activation. Cell. 2014;159:647-61.

21. Cong L, Ran FA, Cox D, Lin S, Barretto R, Habib N, et al. Multiplex genome engineering using CRISPR/Cas systems. Science. 2013;339:819-23.

22. Konermann S, Brigham MD, Trevino AE, Joung J, Abudayyeh OO, Barcena $\mathrm{C}$, et al. Genome-scale transcriptional activation by an engineered CRISPR-Cas9 complex. Nature. 2015;517:583-8.

23. Mandal P, Berger SB, Pillay S, Moriwaki K, Huang C, Guo H, et al. RIP3 induces apoptosis independent of pronecrotic kinase activity. Mol Cell. 2014;56:481-95.

24. Krepler C, Chunduru SK, Halloran MB, He X, Xiao M, Vultur A, et al. The novel SMAC mimetic birinapant exhibits potent activity against human melanoma cells. Clin Cancer Res. 2013;19:1784-94.

25. Dondelinger Y, Hulpiau P, Saeys Y, Bertrand MJM, Vandenabeele P. An evolutionary perspective on the necroptotic pathway. Trends Cell Biol. 2016;26:721-32.

26. Degterev A, Maki JL, Yuan J. Activity and specificity of necrostatin-1, small-molecule inhibitor of RIP1 kinase. Cell Death Differ. 2013;20:366.

27. Callow MG, Watanabe C, Wickliffe KE, Bainer R, Kummerfield S, Weng J, et al. CRISPR whole-genome screening identifies new necroptosis regulators and RIPK1 alternative splicing. Cell Death Dis. 2018;9:261.

28. Taylor KM, Hiscox S, Nicholson RI, Hogstrand C, Kille P. Protein kinase CK2 triggers cytosolic zinc signaling pathways by phosphorylation of zinc channel ZIP7. Sci Signal. 2012;5:ra11.

29. Yan G, Zhang Y, Yu J, Yu Y, Zhang F, Zhang Z, et al. Slc39a7/ zip7 plays a critical role in development and zinc homeostasis in zebrafish. PLoS ONE. 2012;7:e42939.

30. Ohashi W, Kimura S, Iwanaga T, Furusawa Y, Irie T, Izumi H, et al. Zinc transporter SLC39A7/ZIP7 promotes intestinal epithelial self-renewal by resolving ER stress. PLoS Genet. 2016;12: e1006349.

31. Bin BH, Bhin J, Seo J, Kim SY, Lee E, Park K, et al. Requirement of zinc transporter SLC39A7/ZIP7 for dermal development to fine-tune endoplasmic reticulum function by regulating protein disulfide isomerase. J Invest Dermatol. 2017;137:1682-91.

32. Taylor KM, Morgan HE, Johnson A, Nicholson RI. Structurefunction analysis of HKE4, a member of the new LIV-1 subfamily of zinc transporters. Biochem J. 2004;377:131-9.

33. Hart T, Chandrashekhar M, Aregger M, Steinhart Z, Brown KR, MacLeod G, et al. High-resolution CRISPR screens reveal fitness genes and genotype-specific cancer liabilities. Cell. 2015;163: 1515-26.

34. Tzelepis K, Koike-Yusa H, De Braekeleer E, Li Y, Metzakopian E, Dovey OM, et al. A CRISPR dropout screen identifies genetic vulnerabilities and therapeutic targets in acute myeloid leukemia. Cell Rep. 2016;17:1193-205.
35. Blomen VA, Majek P, Jae LT, Bigenzahn JW, Nieuwenhuis J, Staring J, et al. Gene essentiality and synthetic lethality in haploid human cells. Science. 2015;350:1092-6.

36. Groth C, Sasamura T, Khanna MR, Whitley M, Fortini ME. Protein trafficking abnormalities in Drosophila tissues with impaired activity of the ZIP7 zinc transporter Catsup. Development. 2013;140:3018-27.

37. Braakman I, Bulleid NJ. Protein folding and modification in the mammalian endoplasmic reticulum. Annu Rev Biochem. 2011;80: 71-99.

38. Freeze HH, Kranz C. Endoglycosidase and glycoamidase release of N-linked glycans. Curr Protoc Mol Biol. 2010;Unit17:3A.

39. Burckstummer T, Banning C, Hainzl P, Schobesberger R, Kerzendorfer C, Pauler FM, et al. A reversible gene trap collection empowers haploid genetics in human cells. Nat Methods. 2013;10:965-71.

40. Kambe T, Tsuji T, Hashimoto A, Itsumura N. The physiological, biochemical, and molecular roles of zinc transporters in zinc homeostasis and metabolism. Physiol Rev. 2015;95:749-84.

41. Zhang T, Liu J, Fellner M, Zhang C, Sui D, Hu J. Crystal structures of a ZIP zinc transporter reveal a binuclear metal center in the transport pathway. Sci Adv. 2017;3:e1700344.

42. Ramirez VP, Gurevich I, Aneskievich BJ. Emerging roles for TNIP1 in regulating post-receptor signaling. Cytokine Growth Factor Rev. 2012;23:109-18.

43. Verstrepen L, Carpentier I, Beyaert R. The biology of A20binding inhibitors of NF-kappaB activation (ABINs). Adv Exp Med Biol. 2014;809:13-31.

44. Heyninck K, Kreike MM, Beyaert R. Structure-function analysis of the A20-binding inhibitor of NF-kappa B activation, ABIN-1. FEBS Lett. 2003;536:135-40.

45. Oshima S, Turer EE, Callahan JA, Chai S, Advincula R, Barrera J, et al. ABIN-1 is a ubiquitin sensor that restricts cell death and sustains embryonic development. Nature. 2009;457:906-9.

46. Nanda SK, Lopez-Pelaez M, Arthur JS, Marchesi F, Cohen P. Suppression of IRAK1 or IRAK4 catalytic activity, but not type 1 IFN signaling, prevents lupus nephritis in mice expressing a ubiquitin binding-defective mutant of ABIN1. J Immunol. 2016;197:4266-73.

47. Nanda SK, Venigalla RK, Ordureau A, Patterson-Kane JC, Powell DW, Toth R, et al. Polyubiquitin binding to ABIN1 is required to prevent autoimmunity. J Exp Med. 2011;208:1215-28.

48. Zhou J, Wu R, High AA, Slaughter CA, Finkelstein D, Rehg JE, et al. A20-binding inhibitor of NF-kappaB (ABIN1) controls Tolllike receptor-mediated CCAAT/enhancer-binding protein beta activation and protects from inflammatory disease. Proc Natl Acad Sci USA. 2011;108:E998-1006.

49. Ippagunta SK, Gangwar R, Finkelstein D, Vogel P, Pelletier S, Gingras S, et al. Keratinocytes contribute intrinsically to psoriasis upon loss of Tnip1 function. Proc Natl Acad Sci USA. 2016;113: E6162-E71.

50. Darding M, Feltham R, Tenev T, Bianchi K, Benetatos C, Silke J, et al. Molecular determinants of Smac mimetic induced degradation of cIAP1 and cIAP2. Cell Death Differ. 2011;18:1376-86.

51. Holler N, Zaru R, Micheau O, Thome M, Attinger A, Valitutti S, et al. Fas triggers an alternative, caspase-8-independent cell death pathway using the kinase RIP as effector molecule. Nat Immunol. 2000;1:489-95.

52. Brumatti G, Ma C, Lalaoui N, Nguyen NY, Navarro M, Tanzer MC, et al. The caspase- 8 inhibitor emricasan combines with the SMAC mimetic birinapant to induce necroptosis and treat acute myeloid leukemia. Sci Transl Med. 2016;8:339ra69.

53. McComb S, Aguade-Gorgorio J, Harder L, Marovca B, Cario G, Eckert C, et al. Activation of concurrent apoptosis and necroptosis by SMAC mimetics for the treatment of refractory and relapsed ALL. Sci Transl Med. 2016;8:339ra70. 
54. Vince JE, Wong WW, Khan N, Feltham R, Chau D, Ahmed AU, et al. IAP antagonists target cIAP1 to induce TNFalpha-dependent apoptosis. Cell. 2007;131:682-93.

55. Varfolomeev E, Blankenship JW, Wayson SM, Fedorova AV, Kayagaki N, Garg P, et al. IAP antagonists induce autoubiquitination of c-IAPs, NF-kappaB activation, and TNFalphadependent apoptosis. Cell. 2007;131:669-81.

56. Gaither A, Porter D, Yao Y, Borawski J, Yang G, Donovan J, et al. A Smac mimetic rescue screen reveals roles for inhibitor of apoptosis proteins in tumor necrosis factor-alpha signaling. Cancer Res. 2007;67:11493-8.

57. Petersen SL, Wang L, Yalcin-Chin A, Li L, Peyton M, Minna J, et al. Autocrine TNFalpha signaling renders human cancer cells susceptible to Smac-mimetic-induced apoptosis. Cancer Cell. 2007;12:445-56.

58. Grell M, Douni E, Wajant H, Lohden M, Clauss M, Maxeiner B, et al. The transmembrane form of tumor necrosis factor is the prime activating ligand of the $80 \mathrm{kDa}$ tumor necrosis factor receptor. Cell. 1995;83:793-802.

59. Wajant H, Pfizenmaier K, Scheurich P. Tumor necrosis factor signaling. Cell Death Differ. 2003;10:45-65.

60. Siegmund D, Kums J, Ehrenschwender M, Wajant H. Activation of TNFR2 sensitizes macrophages for TNFR1-mediated necroptosis. Cell Death Dis. 2016;7:e2375.

61. Tsai EY, Falvo JV, Tsytsykova AV, Barczak AK, Reimold AM, Glimcher LH, et al. A lipopolysaccharide-specific enhancer complex involving Ets, Elk-1, Sp1, and CREB binding protein and p300 is recruited to the tumor necrosis factor alpha promoter In Vivo. Mol Cell Biol. 2000;20:6084-94.

62. Falvo JV, Uglialoro AM, Brinkman BM, Merika M, Parekh BS, Tsai EY, et al. Stimulus-specific assembly of enhancer complexes on the tumor necrosis factor alpha gene promoter. Mol Cell Biol. 2000;20:2239-47.

63. Yang C, Li J, Yu L, Zhang Z, Xu F, Jiang L, et al. Regulation of RIP3 by the transcription factor $\mathrm{Sp} 1$ and the epigenetic regulator UHRF1 modulates cancer cell necroptosis. Cell Death Dis. 2017;8:e3084.

64. Cesar-Razquin A, Snijder B, Frappier-Brinton T, Isserlin R, Gyimesi G, Bai X, et al. A call for systematic research on solute carriers. Cell. 2015;162:478-87.

65. Lin L, Yee SW, Kim RB, Giacomini KM. SLC transporters as therapeutic targets: emerging opportunities. Nat Rev Drug Discov. 2015;14:543-60.

66. Rebsamen M, Pochini L, Stasyk T, de Araujo ME, Galluccio M, Kandasamy RK, et al. SLC38A9 is a component of the lysosomal amino acid sensing machinery that controls mTORC1. Nature. 2015;519:477-81.
67. Fleisher TA, Oliveira JB. Monogenic defects in lymphocyte apoptosis. Curr Opin Allergy Clin Immunol. 2012;12:609-15.

68. Stojanov S, McDermott MF. The tumour necrosis factor receptorassociated periodic syndrome: current concepts. Expert Rev Mol Med. 2005;7:1-18.

69. Lobito AA, Kimberley FC, Muppidi JR, Komarow H, Jackson AJ, Hull KM, et al. Abnormal disulfide-linked oligomerization results in ER retention and altered signaling by TNFR1 mutants in TNFR1-associated periodic fever syndrome (TRAPS). Blood. 2006;108:1320-7.

70. Schubert D, Klein MC, Hassdenteufel S, Caballero-Oteyza A, Yang L, Proietti M, et al. Plasma cell deficiency in human subjects with heterozygous mutations in Sec61 translocon alpha 1 subunit (SEC61A1). J Allergy Clin Immunol. 2017;141 (4):1427-1438.

71. Wullaert A, Wielockx B, Van Huffel S, Bogaert V, De Geest B, Papeleu $\mathrm{P}$, et al. Adenoviral gene transfer of ABIN-1 protects mice from $\mathrm{TNF} /$ galactosamine-induced acute liver failure and lethality. Hepatology. 2005;42:381-9.

72. Dziedzic SA, Su Z, Jean Barrett V, Najafov A, Mookhtiar AK, Amin P, et al. ABIN-1 regulates RIPK1 activation by linking Met1 ubiquitylation with Lys63 deubiquitylation in TNF-RSC. Nat Cell Biol. 2018;20:58-68.

73. Shalem O, Sanjana NE, Hartenian E, Shi X, Scott DA, Mikkelson $\mathrm{T}$, et al. Genome-scale CRISPR-Cas9 knockout screening in human cells. Science. 2014;343:84-7.

74. Jae LT, Raaben M, Herbert AS, Kuehne AI, Wirchnianski AS, Soh TK, et al. Virus entry. Lassa virus entry requires a triggerinduced receptor switch. Science. 2014;344:1506-10.

75. Sedlyarov V, Eichner R, Girardi E, Essletzbichler P, Goldmann U, Nunes-Hasler P, et al. The bicarbonate transporter SLC4A7 plays a key role in macrophage phagosome acidification. Cell Host Microbe. 2018;23:766-74. e5

76. Sanjana NE, Shalem O, Zhang F. Improved vectors and genomewide libraries for CRISPR screening. Nat Methods. 2014;11:783-4.

77. Love MI, Huber W, Anders S. Moderated estimation of fold change and dispersion for RNA-seq data with DESeq2. Genome Biol. 2014;15:550.

78. Subramanian A, Tamayo P, Mootha VK, Mukherjee S, Ebert BL, Gillette MA, et al. Gene set enrichment analysis: a knowledgebased approach for interpreting genome-wide expression profiles. Proc Natl Acad Sci USA. 2005;102:15545-50.

79. Bordier C. Phase separation of integral membrane proteins in Triton X-114 solution. J Biol Chem. 1981;256:1604-7.

80. Wisniewski JR, Zougman A, Nagaraj N, Mann M. Universal sample preparation method for proteome analysis. Nat Methods. 2009;6:359-62. 